\title{
NURBS-Enhanced Finite Element Method (NEFEM)
}

\author{
A Seamless Bridge Between CAD and FEM
}

\author{
Ruben Sevilla • Sonia Fernández-Méndez • \\ Antonio Huerta
}

Published online: 13 October 2011

(C) CIMNE, Barcelona, Spain 2011

\begin{abstract}
The development of NURBS-Enhanced Finite Element Method (NEFEM) is revisited. This technique allows a seamless integration of the CAD boundary representation of the domain and the finite element method (FEM). The importance of the geometrical model in finite element simulations is addressed and the benefits and potential of NEFEM are discussed and compared with respect to other curved finite element techniques.
\end{abstract}

\section{Introduction}

This paper revisits the state of the art of an efficient methodology to integrate the NURBS boundary representation of the domain into a standard finite element framework: the socalled NURBS-enhanced finite element method (NEFEM).

Non-uniform rational B-splines (NURBS, see [72]) are nowadays widely used for geometric description in Computer Aided Design (CAD). Other popular options for geometric description in CAD are polynomial B-splines (a particular case of NURBS) and subdivision surfaces. This fact

R. Sevilla $(\varangle)$

Civil and Computational Engineering Centre $\left(\mathrm{C}^{2} \mathrm{EC}\right)$,

College of Engineering, Swansea University, Singleton Park,

Swansea, SA2 8PP, Wales, UK

e-mail: R.Sevilla@swansea.ac.uk

S. Fernández-Méndez · A. Huerta

Laboratori de Càlcul Numèric (LaCàN), Departament

de Matemàtica Aplicada III, Universitat Politècnica de Catalunya,

Jordi Girona 1-3, Barcelona, 08034, Spain

S. Fernández-Méndez

e-mail: sonia.fernandez@upc.edu

A. Huerta

e-mail: antonio.huerta@upc.edu has motivated the development of novel numerical techniques considering CAD descriptions of the computational domain.

NURBS-Enhanced Finite Element Method uses NURBS to accurately describe the boundary of the computational domain and the solution is approximated using polynomials defined with Cartesian coordinates, directly in the physical space. From a practical point of view, NEFEM considers efficient strategies for numerical integration on elements affected by curved boundaries. It is worth remarking that at elements not intersecting the boundary classical finite elements (FEs) are used, preserving the efficiency of the finite element method (FEM).

NEFEM was first presented for 2D domains in [79], showing the advantages in front of classical isoparametric FEs using both continuous and discontinuous Galerkin formulations for the numerical solution of some test problems. It is important to remark that all the ideas presented in [79] are valid not only when the boundary of the domain is parametrized by NURBS, but for any piecewise boundary parametrization. The discussion is centered on NURBS boundary parametrization because they are the most extended technology in CAD. In [81] NEFEM was shown to be a powerful tool for solving compressible flow problems governed by the Euler equations of gas dynamics.

Several high-order FE methodologies for the treatment of curved boundaries are discussed and compared in [83], including isoparametric FEM, Cartesian FEM, $p$-FEM and NEFEM. Numerical examples show that NEFEM is not only more accurate than FE methods with an approximate boundary representation, but also outperforms $p$-FEM with an exact boundary representation, showing the advantages of combining Cartesian approximation with exact boundary representation. 
In [82] the extension of NEFEM to $3 \mathrm{D}$ domains is presented. Although conceptually easy, the extension of NEFEM to 3D requires attention to several geometric aspects. The advantages of NEFEM in front of other curved FE techniques are discussed and illustrated using numerical examples.

This paper is organized as follows. In Sect. 2 a review of FE techniques for curved boundaries with a historical perspective is presented. An introductory overview of NonUniform Rational B-Splines is given in Sect. 3. Section 4 is devoted to review NEFEM. The core concept is first described and technical details regarding the polynomial approximation and the numerical integration in curved elements is presented. Optimal a priori error estimates for NEFEM are recalled and, finally, some implementation details are summarized in order to facilitate the adoption of NEFEM by the FE community. In Sect. 5 a critical comparison between FE techniques used in domains with curved boundaries is presented, with particular emphasis in two issues: the exact boundary representation of the domain and the consistency of the approximation. The numerical examples presented in Sect. 6 range from second-order elliptic problems to more complex problems involving the numerical solution of Euler and Maxwell's equations. The application of NEFEM in continuous and discontinuous Galerkin frameworks is illustrated. In the numerical examples NEFEM is shown as a powerful strategy to efficiently treat curved boundaries and to avoid excessive mesh refinement when complex geometric objects are considered. Finally, Sect. 7 summarizes the main conclusions and Sect. 8 discusses some areas of future research.

\section{Historical Perspective}

The origin of the finite element method (FEM) is mainly attributed to pioneer works in the field of airplane structural analysis, see $[19,105]$ for an overview of the early history of the FEM. The need of curved elements to improve the quality of the shape discretization soon arose and the first approach introduced to efficiently deal with curved boundaries were the so-called isoparametric elements, see [35, 104]. In these works the authors pointed out that in a practical setting, mesh refinement is governed by the need to accurately represent curved geometric objects, and the use of curved elements was proposed in order to retain accurate boundary descriptions without performing excessive mesh refinement. The key idea of isoparametric elements was to employ the same polynomial functions to approximate the solution and to define the mapping between the reference element and the physical element, hence the term isoparametric. This approach was rapidly adopted for solid mechanics applications due to its straightforward implementation and its relatively good performance.
During the 1970s, there was an increasing interest in the development and analysis of curved finite elements, see [17, $38,39,77,94,107-109]$. The technique presented in [107, 109] is recognized to be the first FEM considering an exact boundary representation. Triangular elements with one curved edge were introduced, and the isoparametric mapping was modified to map a reference element into the triangular element with an exact boundary description. A similar approach was developed in [77], also using triangular elements with one curved side corresponding to the exact boundary. Alternatives to the standard polynomial approximation of the solution were also proposed within the context of curved FEs with an exact boundary representation, see for instance the rational basis in $[94,95]$. Nevertheless, all these FE techniques with exact boundary representation were not considered a practical tool, but a mathematical idealization, due to the impossibility to extend the ideas to 3D domains.

The necessity of accurate geometric descriptions in the automotive industry was the origin of the so-called blending mappings proposed in [38]. This mappings represented an inflection point in the development of general procedures to exactly treat curved boundaries as they were the core concept of a new and successful family of elements called transfinite elements, see [39]. The key idea was to introduce blending functions to define a mapping between a reference square and a subdomain with the boundary given by four parametric curves. The problem of geometric inaccuracies associated to the isoparametric transformation are removed by blending mappings, and higher degrees of interpolation can be successfully employed. Therefore, blending mappings were naturally adopted in the so-called $p$-version of the FEM ( $p$-FEM), see [4] and a recent review in [87]. In this approach the mesh remain fixed (usually containing large elements) and the degree of the approximations is increased in order to properly capture the solution. Therefore, an accurate geometric description is crucial in this context.

During the 1980s a great effort was dedicated to the study of approximation spaces that guarantee optimal convergence of the finite element method in the presence of curved boundaries, see for instance [12, 14, 32, 61, 96].

Despite the early introduction of curved FE techniques for solid mechanics applications, in the 1980s and 1990s the geometric description was an important handicap for computational fluid dynamics (CFD). In particular, linear approximation of curved walls in numerical solutions of Euler equations of gas dynamics was identified as the origin of spurious entropy production near curved boundaries. In a finite volume (FV) context, see [7], the problem was identified in [8, 27]. In [99] a local approximation of the curvature is used in order to enhance the imposition of the wall boundary condition on curved boundaries, resulting in a dramatic reduction of the entropy production. More recently, in the so-called spectral volume method, see [100], the accurate 
treatment of curved boundaries has been identified as a critical issue. In [98] the spectral volume method is extended to the two-dimensional Euler equations with curved boundaries and in [44] the authors implement the ideas of [55] to enhance the accuracy of the approximation in the presence of curved walls. In a discontinuous Galerkin (DG) context, see [20], the same problematic when solving Euler equations was identified in [9]. In [93] a detailed study of this problem is presented to conclude that accurate results can only be obtained taking into account the curvature of the boundary. More recently, in [55] a new methodology is presented for the computation of the fluxes across curved boundaries but, unfortunately, the proposed method is not conservative. In [34], the advantages of using high-order isoparametric elements for the numerical solution of inviscid compressible flow problems are also illustrated. Using ultra-coarse meshes and high-order approximations the authors show the benefits of curved elements. In fact, this work evidences the necessity of better than isoparametric boundary representation when coarse meshes are considered because the $\mathcal{C}^{0}$ continuity of the curved boundary between elements is shown to have an important impact in quantities of interest such as the pressure coefficient over an airfoil profile. More recently, in [37] a different mapping is proposed based on the boundary representation with Bézier curves. Imposing $\mathcal{C}^{1}$ continuity of the approximated boundary an important improvement is obtained compared to standard isoparametric mappings. It is worth recalling that the necessity of curved elements and a $\mathcal{C}^{1}$ continuity approximation of the boundary in fluid mechanics applications was very early pointed out as a key issue for obtaining accurate solution without excessive mesh refinement, see for instance [68].

Accurate geometric descriptions are also relevant in other areas of fluid mechanics such as the numerical solution of the Navier-Stokes equations, see for instance [57, 62], but the importance of the geometrical model is not exclusive of fluid mechanics. Maxwell's equations are also very sensitive to an accurate geometric description. In [103] the error induced by isoparametric approximations of curvilinear geometries is studied. By solving the 3D Maxwell's equations in a sphere, the authors show that an exact description of the geometry reduces the error in one order of magnitude compared to isoparametric elements. Similar conclusions are derived in [64] for linear elasticity problems. They conclude that sizable errors are present in the numerical solution when the order of the geometric approximation is lower than the order of the functional interpolation. The relevance of an accurate geometric model for some applications in solid mechanics is also illustrated in [69], where the use of B-splines is proposed for the geometric representation of the interface in frictionless contact problems.

Curved FE were developed during the 1970s and 1980s without regarding the emerging Computer Aided Design
(CAD) industry. The great impact of CAD technology in the FE community arrived in the 1980s. In fact, researchers on the field of shape optimization were the first to promote the so-called marriage of CAD and FE. In a shape optimization process, the integration of CAD into the analysis stage is crucial to avoid the geometric approximation inherent in a mesh. In [75] transfinite elements were implemented using NURBS for the geometric description. Obviously, the rational nature of NURBS leads to rational function maps between the reference element and the element in the physical space. Note however that the solution is approximated using polynomials. Some inaccuracies associated to the lack of satisfaction of the isoparametric concept are mentioned in [75], but not further explained. To obtain an isoparametric approach, the exact boundary representation was abandoned, and a simplification of the geometry with polynomial B-Splines was proposed. Thus, some of the advantages of NURBS, such as exact representation of conics, were lost.

Over the 1990s, other authors focused their attention on integrating NURBS technology into FE codes. For instance, in [29] an element geometric mapping also based on blending functions with NURBS is proposed. More recently, in [67], $p$-FEM with NURBS for the boundary representation is applied to plane elasticity problems. The inaccuracies previously mentioned in [75] are also reported, and the lack of satisfaction of the isoparametric concept is alleviated by a rational enrichment of the polynomial basis used to approximate the solution.

The relevance of an accurate geometry description also motivated, in the late 1990s, a new family of FE-like techniques based on CAD, which is still today object of intensive research: isogeometric methods. The key idea is to use the same CAD representation for both geometrical design and analysis. Thus, contrary to classical FE methodologies, the whole domain is treated as a CAD entity, not only the boundary of the domain. Moreover, classical polynomial approximations of the solution are abandoned and the solution is approximated with the same basis used in the CAD environment. The first application is again encountered on shape optimization, using B-Splines for the geometrical description and for the mechanical analysis, see [53]. In the 2000s, more advanced CAD technology have been applied following the same rationale. In [18] subdivision surfaces are used for thin shell analysis and relevant advantages are found due to the sensitivity of shells to an inaccurate geometric representation. More recently, NURBS have been used to develop isogeometric methods, see for instance [49, 51]. In [51] the application of a NURBS isogeometric method to shape optimization processes is presented. Finally, in [49] a more general framework known as isogeometric analysis is proposed. This approach is not only focused on the accurate representation of the geometry, but also in the possibilities of NURBS as a basis for the approximation. See more recent advances in [25]. 
Fig. 5 (a) Parametric space, (b) NURBS surface with knot lines, and (c) control net
Fig. 6 (a) Parametric space trimmed by the thick curve, (b) trimmed NURBS surface with knot lines and the thick curve used to trim the initial surface of Fig. 5, and (c) control net

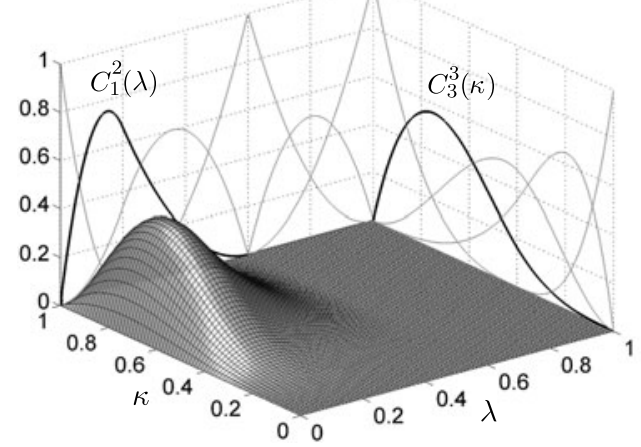

(a) $S_{1,3}^{2,3}(\lambda, \kappa):=C_{1}^{2}(\lambda) C_{3}^{3}(\kappa)$

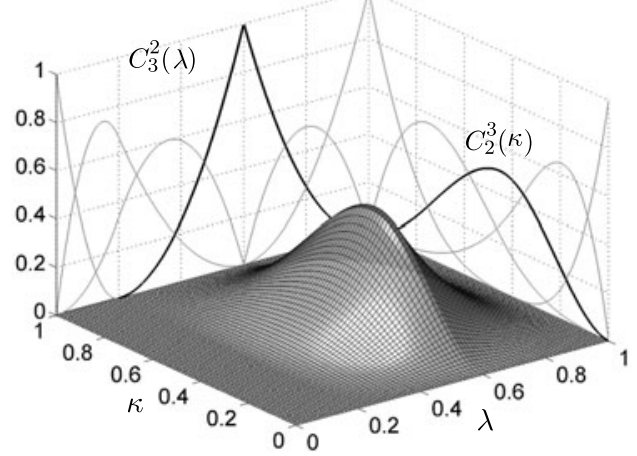

(b) $S_{3,2}^{2,3}(\lambda, \kappa):=C_{3}^{2}(\lambda) C_{2}^{3}(\kappa)$

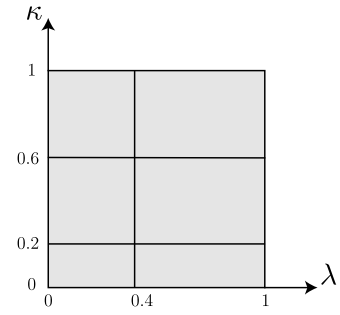

(a)

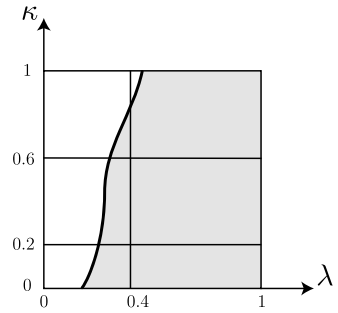

(a)

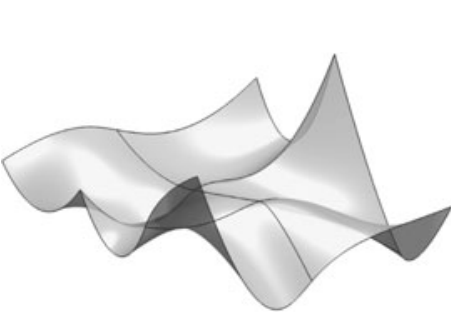

(b)

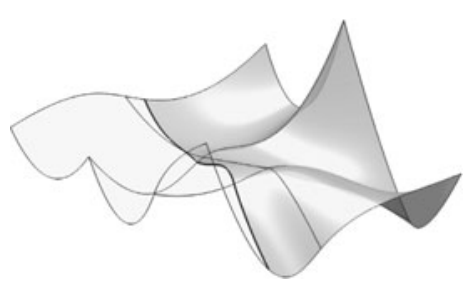

(b)

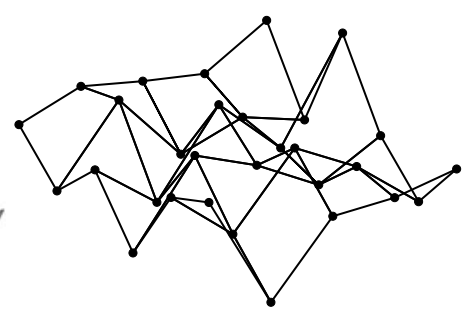

(c)

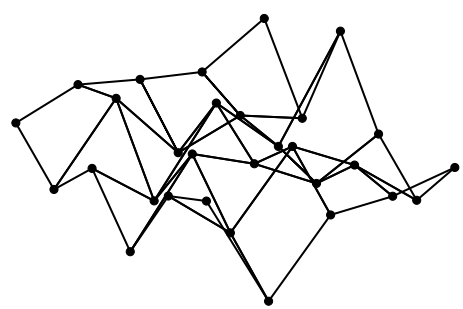

(c)
$\Lambda^{\lambda}=\{0,0,0,0.2,0.6,0.6,1,1,1\}$

$\Lambda^{\kappa}=\{0,0,0,0,0.4,1,1,1,1\}$.

Complete 1D basis are represented for each direction to illustrate the construction of $2 \mathrm{D}$ basis functions as described in (2).

Note that NURBS surfaces change their definition along knot lines, that is when $\lambda=\lambda_{i}$, for $i=1, \ldots, \mathrm{n}_{\mathrm{k}}^{\lambda}$, or $\kappa=\kappa_{i}$, for $i=1, \ldots, \mathrm{n}_{\mathrm{k}}^{\kappa}$. An example of a NURBS surface is represented in Fig. 5 with the corresponding control net. Knot lines are represented on the NURBS surface in order to stress the discontinuous nature of the parametrization.

An example of a trimmed NURBS surface is represented in Fig. 6, showing the NURBS surface of Fig. 5 trimmed with the thick curve. In practical applications, it is also common to deal with singular (or singularly parametrized) NURBS surfaces. Such surfaces contain at least one singular point, defined as a point where a directional derivative is zero. For these surfaces, knot lines typically converge to the singular point, see an example in Fig. 7.

\section{NURBS-Enhanced Finite Element Method (NEFEM)}

This section introduces the fundamental ideas of NEFEM in 2D and 3D domains, see [79, 82] for further details. The core concept is first described and the strategy to define curved entities in NEFEM is detailed. Special attention is paid to the design of efficient strategies in order to define high-order approximations and to perform the numerical integration on curved NEFEM elements. Optimal a priori error estimates for $h$ and $p$ refinement are recalled, and implementation details are given in order to facilitate the adoption of NEFEM.

\subsection{NEFEM Concept}

Consider an open bounded domain $\Omega$ whose boundary $\partial \Omega$, or a portion of it, is parametrized by NURBS curves in $2 \mathrm{D}$ 
Fig. 7 (a) Parametric space,

(b) singular NURBS surface with knot lines, and (c) control net

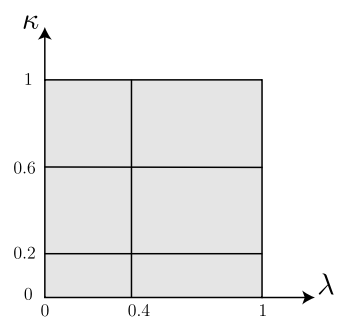

(a)

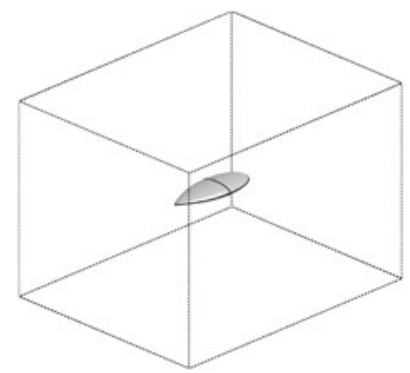

(a)

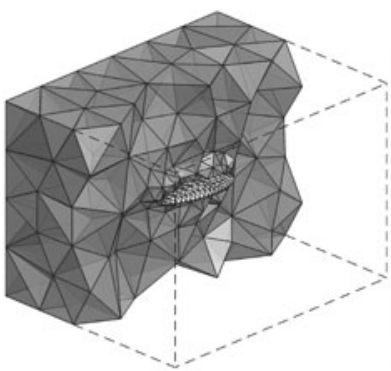

(b)

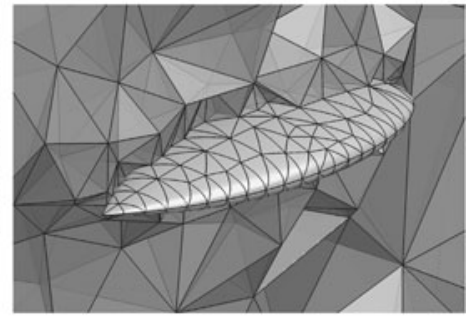

(c) or surfaces in $3 \mathrm{D}$. In $2 \mathrm{D}$ every NURBS is assumed to be parametrized by

$$
\boldsymbol{C}:[0,1] \longrightarrow \boldsymbol{C}([0,1]) \subseteq \partial \Omega \subset \mathbb{R}^{2} .
$$

Analogously, in 3D every NURBS is assumed to be parametrized by

$\boldsymbol{S}:[0,1]^{2} \longrightarrow \boldsymbol{S}\left([0,1]^{2}\right) \subseteq \partial \Omega \subset \mathbb{R}^{3}$

A regular partition of the domain $\bar{\Omega}=\bigcup_{e} \bar{\Omega}_{e}$ in simplices is assumed, such that $\Omega_{i} \cap \Omega_{j}=\emptyset$, for $i \neq j$. For instance, Fig. 8 shows a 3D computational domain with part of the boundary defined by NURBS surfaces corresponding to the NASA almond [30], a useful geometry for benchmarking electromagnetic scattering codes. A cut through an unstructured tetrahedral mesh is also represented in Fig. 8, including the surface triangular mesh on the almond.

As usual in FE mesh generation codes, it is assumed that every curved boundary face belongs to a unique NURBS. That is, one element face can not be defined by portions of two, or more, different NURBS. Note however that the piecewise definition of each NURBS is independent on the mesh discretization. Thus, NURBS parametrization can change its definition within one face, that is, FE edges do not need to coincide with knot lines. Figure 9 shows the image of the knot lines of the NASA almond surfaces and the surface triangulation corresponding to the mesh represented in Fig. 8. It can be observed that spatial discretization is independent of the piecewise NURBS surface parametrization. It is worth remarking that allowing changes of NURBS parametrization to be independent on the spatial discretization means that special attention must to be paid to the numerical integration over elements affected by the NURBS boundary representation, see Sect. 4.4.2.

An element without any edge or face in contact with NURBS boundaries has planar faces and it is defined and treated as a standard FE. Therefore, in the vast majority of the domain, interpolation and numerical integration are standard, preserving the computational efficiency of classical FEM. Specifical numerical strategies for interpolation and numerical integration are needed only for those elements affected by NURBS boundaries.

\subsection{Curved Elements}

In NEFEM, curved elements are defined in terms of the NURBS boundary representation of the domain. The formal definition of curved faces and elements in a NEFEM mesh is given in this section.

\subsubsection{D Elements}

Let $\Gamma_{e}$ be an edge on the NURBS boundary parametrized by $\boldsymbol{C}$, and $\boldsymbol{x}_{1}, \boldsymbol{x}_{2} \in \partial \Omega$ the two vertices on the NURBS boundary, see Fig. 10. The edge is defined by

$\Gamma_{e}:=\boldsymbol{C}\left(\left[\lambda_{1}^{e}, \lambda_{2}^{e}\right]\right)$,

where $\lambda_{1}^{e}$ and $\lambda_{2}^{e}$ are the parametric coordinates (in the parametric space of the NURBS) of the end points of $\Gamma_{e}$.

A curved triangular element with an edge on the NURBS boundary is defined as a convex linear combination of the 
Fig. 9 (a) Knot lines of the

NURBS surfaces defining the NASA almond, (b) surface triangulation, and (c) surface triangulation and knot lines

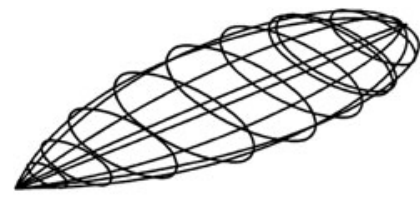

(a)
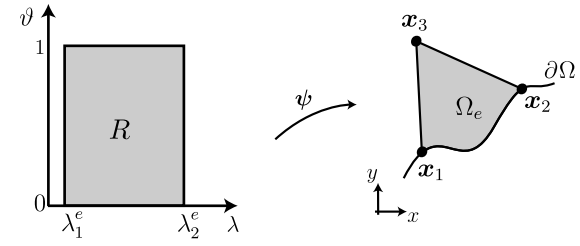

Fig. 10 Parametrization of a curved triangular element with a edge on the NURBS boundary

curved NURBS edge and the interior vertex. For instance, element represented in Fig. 10 is parametrized by

$\psi: R \longrightarrow \Omega_{e}$

$(\lambda, \vartheta) \longmapsto \psi(\lambda, \vartheta):=(1-\vartheta) C(\lambda)+\vartheta x_{3}$,

where $R=\left[\lambda_{1}^{e}, \lambda_{2}^{e}\right] \times[0,1]$ and $\boldsymbol{x}_{3}$ is the internal vertex of $\Omega_{e}$, see Fig. 10 .

\subsubsection{D Elements}

Let $\Upsilon_{e}$ be a tetrahedral face on the NURBS boundary parametrized by $\boldsymbol{S}$, and $\boldsymbol{x}_{1}, \boldsymbol{x}_{2}, \boldsymbol{x}_{3} \in \partial \Omega$ the three vertices on the NURBS boundary, see Fig. 11. Assuming that the vertices $\boldsymbol{x}_{1}, \boldsymbol{x}_{2}, \boldsymbol{x}_{3}$ do not correspond to singular points of the NURBS parametrization, a straight-sided triangle $\Lambda_{e}$ in the parametric space of the NURBS is uniquely defined by the parametric coordinates of the vertices, $S^{-1}\left(x_{1}\right), S^{-1}\left(x_{2}\right)$ and $S^{-1}\left(x_{3}\right)$. The curved face with a NURBS boundary representation, $\Upsilon_{e}$, is defined as the image of the straight-sided triangle $\Lambda_{e}$ by the NURBS parametrization $S$,

$\Upsilon_{e}:=S\left(\Lambda_{e}\right)$

as illustrated in Fig. 11.

Note that when the surface $S$ is trimmed by a curve $C$ in the parametric space of the NURBS, the edges of the triangle $\Lambda_{e}$ must be replaced by trimmed NURBS curves. In such cases $\Lambda_{e}$ is a curved triangle in the parametric space of the NURBS and curved edges of $\Lambda_{e}$ are NURBS curves (used to trim the original surface), see an example in Fig. 12. Finally, assuming that one of the vertices of the tetrahedral face corresponds to a singular point of the NURBS parametrization, $\Lambda_{e}$ must be defined as a quadrilateral in the parametric space of the NURBS, see an example in Fig. 13.

Interior curved faces with an edge on the NURBS boundary are defined as a convex linear combination of the curved edge and the interior face node. For instance, curved face $\Upsilon_{e}^{E}$ represented in Fig. 11 is parametrized by

$$
\begin{gathered}
\boldsymbol{\Theta}_{x_{4}}:\left[\varrho_{1}, \varrho_{2}\right] \times[0,1] \longrightarrow \Upsilon_{e}^{E} \\
(\varrho, \sigma) \longmapsto \boldsymbol{\Theta}_{x_{4}}(\varrho, \sigma) \\
:=(1-\sigma) \boldsymbol{\theta}(\varrho)+\sigma \boldsymbol{x}_{4},
\end{gathered}
$$

where $\boldsymbol{\theta}\left(\left[\varrho_{1}, \varrho_{2}\right]\right)$ parametrizes the curved edge from vertex $\boldsymbol{x}_{2}$ to vertex $\boldsymbol{x}_{3}$. Note that this approach to define interior curved faces ensures the same definition of an interior curved face as seen from the two elements sharing this face. Note also that other types of curved faces are present in real meshes, such as faces with several edges over the NURBS boundary. A systematic way of defining all possible curved faces is presented in Sect. 4.4.2.

With this definition of curved faces, a curved tetrahedral element with a face on the NURBS boundary is defined by a convex linear combination of the curved NURBS face and the interior vertex. For instance, element represented in Fig. 11 is parametrized by

$$
\begin{aligned}
& \boldsymbol{\Psi}: \Lambda_{e} \times[0,1] \longrightarrow \Omega_{e} \\
&(\lambda, \kappa, \vartheta) \longmapsto \boldsymbol{\Psi}(\lambda, \kappa, \vartheta) \\
&:=(1-\vartheta) \boldsymbol{S}(\lambda, \kappa)+\vartheta \boldsymbol{x}_{4},
\end{aligned}
$$

where $\boldsymbol{x}_{4}$ denotes the interior vertex of $\Omega_{e}$. Similarly, an element with an edge on the NURBS boundary corresponds to a convex linear combination of one of its curved faces an the opposite node, and can be parametrized by

$$
\begin{aligned}
& \boldsymbol{\Phi}:\left[\varrho_{1}, \varrho_{2}\right] \times[0,1]^{2} \longrightarrow \Omega_{e} \\
&(\varrho, \sigma, \tau) \longmapsto \boldsymbol{\Phi}(\varrho, \sigma, \tau) \\
&:=(1-\tau) \boldsymbol{\Theta}_{x_{3}}(\varrho, \sigma)+\tau \boldsymbol{x}_{4}
\end{aligned}
$$

where $\boldsymbol{x}_{3}$ and $\boldsymbol{x}_{4}$ are the interior vertices of $\Omega_{e}$, see Fig. 14 . Note that the definition of $\boldsymbol{\Phi}$ in (7) is independent on the order of the interior vertices $\boldsymbol{x}_{3}$ and $\boldsymbol{x}_{4}$. That is, element $\Omega_{e}$ can be equivalently parametrized by

$\boldsymbol{\Phi}(\varrho, \sigma, \tau):=(1-\tau) \boldsymbol{\Theta}_{\boldsymbol{x}_{4}}(\varrho, \sigma)+\tau \boldsymbol{x}_{3}$.

It is worth remarking that interior edges, (i.e., edges with no more than one node over the NURBS boundary) are considered as straight edges. Note that this assumption allows 
Fig. 11 Parametrization of a curved tetrahedral element with a face on the NURBS boundary, showing a face $\Upsilon_{e}$ on the NURBS boundary, and a face NURBS boundary, $\Upsilon_{e}^{E}$ $\Upsilon_{e}^{E}$ with an edge on the

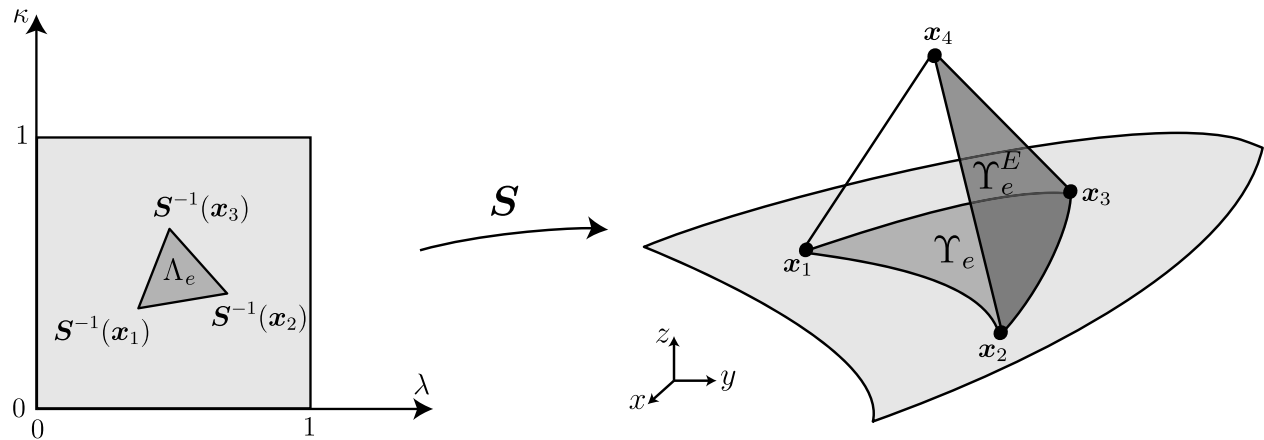

Fig. 12 Curved tetrahedral face on a trimmed NURBS

boundary. NURBS surface $S$ is trimmed by NURBS curve $\boldsymbol{C}$, leading to a curved triangle $\Lambda_{e}$ in the parametric space

Fig. 13 Curved tetrahedral face on a singular NURBS boundary with a singular point, leading to a quadrilateral $\Lambda_{e}$ in the parametric space
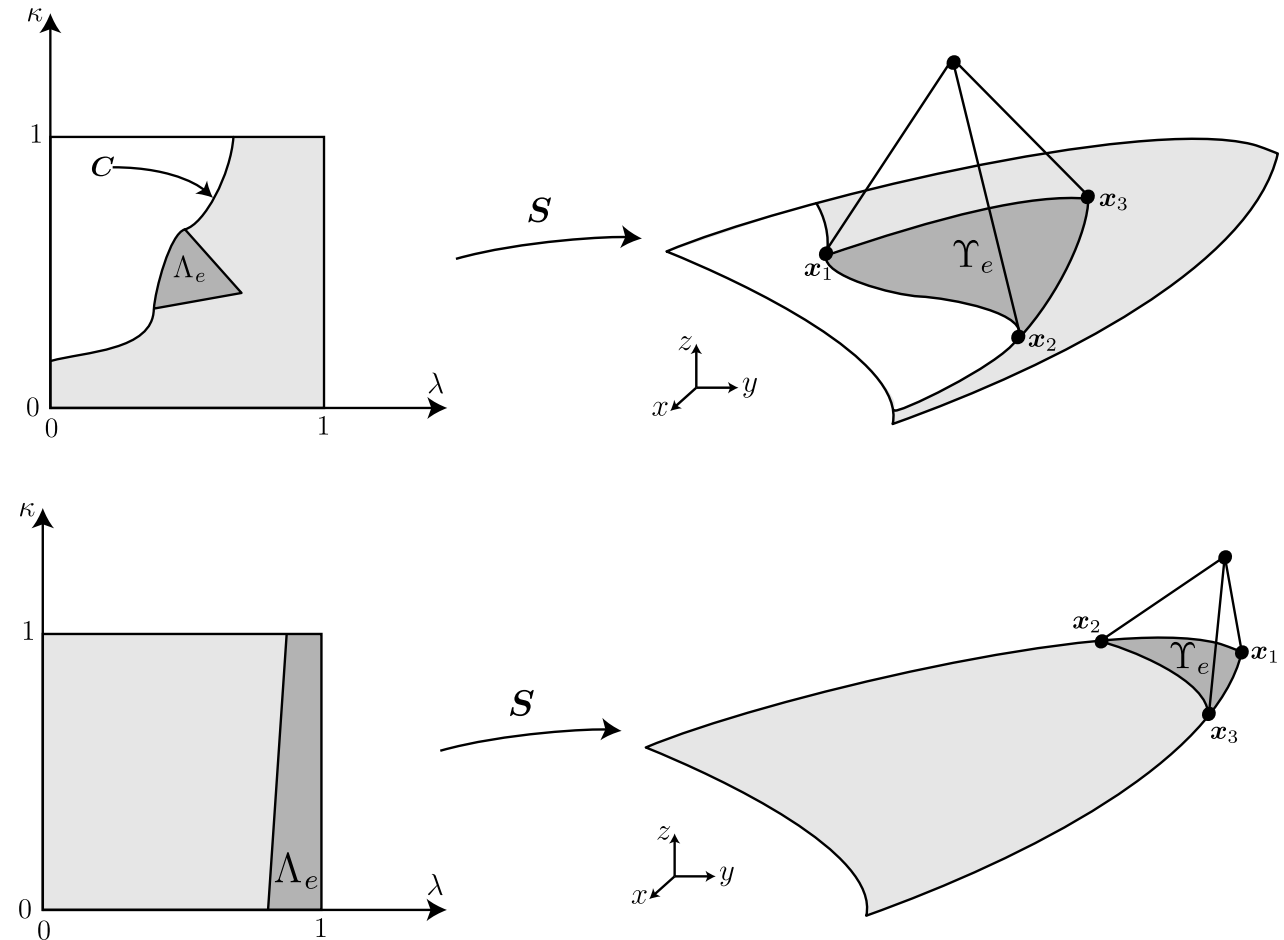

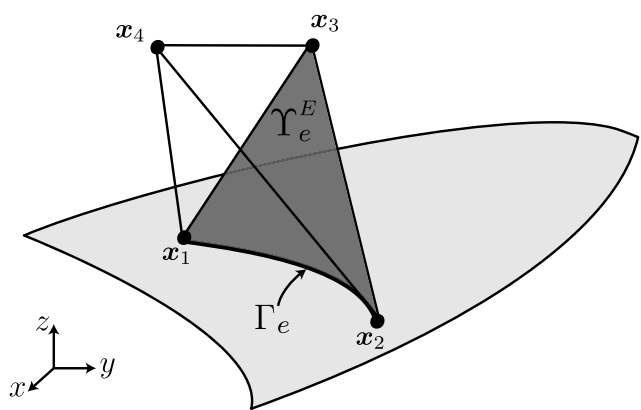

Fig. 14 Curved tetrahedral elements with an edge on the NURBS boundary

to ensure that the elements affected by the NURBS boundary representation of the domain are only elements with at least one face or one edge over the NURBS boundary, and therefore, the overhead introduced by NEFEM is restricted to a very small portion of the total number of elements.

\subsection{High-Order Approximation in Curved Elements}

NEFEM considers nodal polynomial interpolation in each element. To ensure reproducibility of polynomials in the physical space, NEFEM defines the approximation directly with Cartesian coordinates, $\boldsymbol{x}$, that is

$u(\boldsymbol{x}) \simeq u^{h}(\boldsymbol{x})=\sum_{i=1}^{\mathrm{n}_{\mathrm{en}}} u_{i} N_{i}(\boldsymbol{x})$,

where $u_{i}$ are nodal values, $N_{i}$ are polynomial shape functions (Lagrange polynomials) of order $p$ in $\boldsymbol{x}$, and $\mathrm{n}_{\mathrm{en}}$ is the number of element nodes. Recall that in isoparametric FEM or $p$-FEM the approximation is defined in a reference element. However, contrary to NEFEM, the definition of the polynomial basis for high-order curved elements does not ensure reproducibility of polynomials in the physical space.

To make the computation of Lagrange polynomial basis more systematic, for any degree and for any distribution of 
cal computation of the integral, namely

$\int_{\Gamma_{e}} f d \ell \approx \sum_{i=1}^{\mathrm{n}_{\mathrm{ip}}} f\left(\boldsymbol{C}\left(\lambda_{i}\right)\right)\left\|J_{\boldsymbol{C}}\left(\lambda_{i}\right)\right\| \omega_{i}$

where $\lambda_{i}$ and $\omega_{i}$ are the coordinates and weights of the $n_{i p}$ integration points in $\left[\lambda_{1}^{e}, \lambda_{2}^{e}\right]$.

Recall that a NURBS parametrization, $\boldsymbol{C}$, is a piecewise rational function whose definition changes at the breakpoints. Thus, an independent numerical quadrature must be considered for each one of the intervals between breakpoints in order to take into account the discontinuous nature of the parametrization.

When a polynomial interpolation of degree $p$ is considered in the NEFEM context, it is interesting to know the minimum number of integration points needed to integrate all the polynomials of degree less or equal to $2 p$ with a desired accuracy. Figure 22 shows the number of integration points needed to integrate all the polynomials of a degree less or equal to $2 p$ with an accuracy of $10^{-6}$ and $10^{-10}$ respectively along the trimmed NURBS describing a quarter of a circle. The results using simple and composite Gauss-Legendre quadratures are displayed. For a NE-

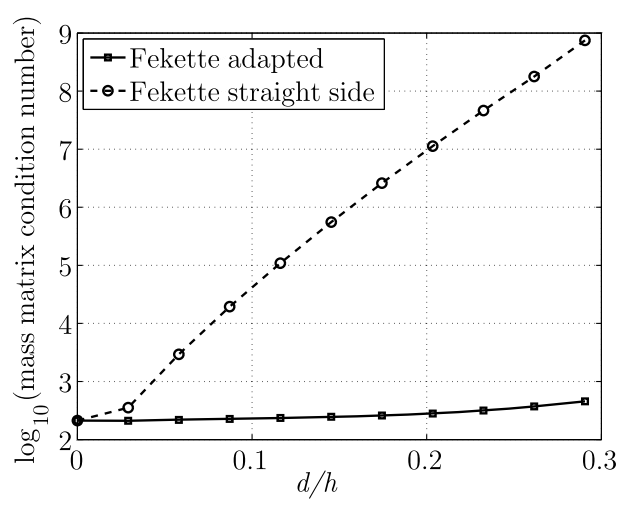

Fig. 21 Condition number of the elemental mass matrix as a function of $d / h$
FEM computation with polynomials of degree $p=5$, simple Gauss-Legendre quadratures provide an accuracy of $10^{-6}$ in the boundary integrals using 10 integration points. GaussLegendre composite quadratures with $n=4$ require 5 subintervals, i.e. 20 integration points, to obtain the same accuracy, and Gauss-Legendre composite quadratures with $\mathrm{n}=8$ require 2 subintervals, i.e. 16 points. For a NEFEM computation with polynomials of degree 10 , an accuracy of $10^{-6}$ is attained with simple Gauss-Legendre quadratures with 15 integration points whereas composite Gauss-Legendre quadratures with $n=4$ require 28 integration points and composite Gauss-Legendre quadratures with $\mathrm{n}=8$ require 24 integration points. If the desired accuracy is $10^{-10}$ the number of integration points is only slightly increased for simple quadratures whereas composite quadrature suffer from a higher increase in computation cost. For instance, for a NEFEM computation with polynomials of degree 10 , simple Gauss-Legendre quadrature require 19 points, composite quadratures with $n=4$ require 16 subintervals, i.e. 64 points, and composite quadratures with $\mathrm{n}=8$ require 4 subintervals, i.e. 32 points. Same conclusions are obtained for the integration along other trimmed NURBS, see [80].

Although the faster convergence is obtained for highorder simple quadratures, the use of composite rules is very attractive, allowing to control the error in a straightforward manner, see for instance [28]. Numerical experiments reveal that other popular quadrature rules such as trapezoidal and Simpson composite rules or Romberg's integration are not competitive compared to Gauss-Legendre quadrature rules.

NEFEM also requires to compute integrals in an element $\Omega_{e}$ with one edge $\Gamma_{e}$ on the NURBS boundary, see Fig. 10. Using the transformation in (3), element integrals are computed as

$\int_{\Omega_{e}} f d \Omega=\int_{R} f(\psi(\lambda, \vartheta))\left|J_{\psi}(\lambda, \vartheta)\right| d \lambda d \vartheta$

where $\left|J_{\Psi}\right|$ is the determinant of the Jacobian of the transformation $\psi$. The integral can be evaluated using 1D Gauss-
Fig. 22 Number of integration points required to integrate all the polynomials of degree of equal to $2 p$ with an accuracy of (a) $10^{-6}$ and (b) $10^{-10}$ along the trimmed NURBS describing a quarter of a circle

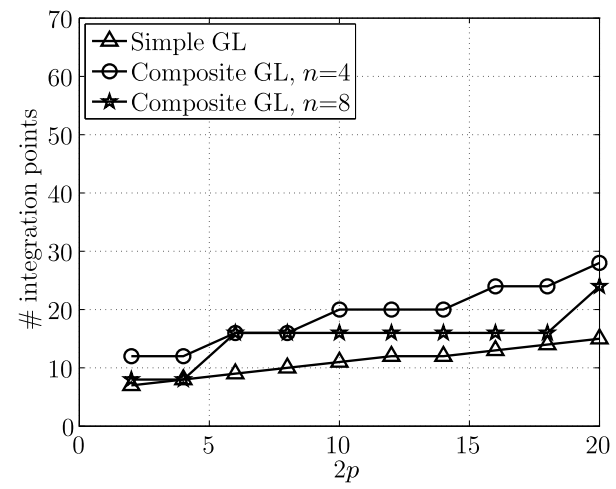

(a)

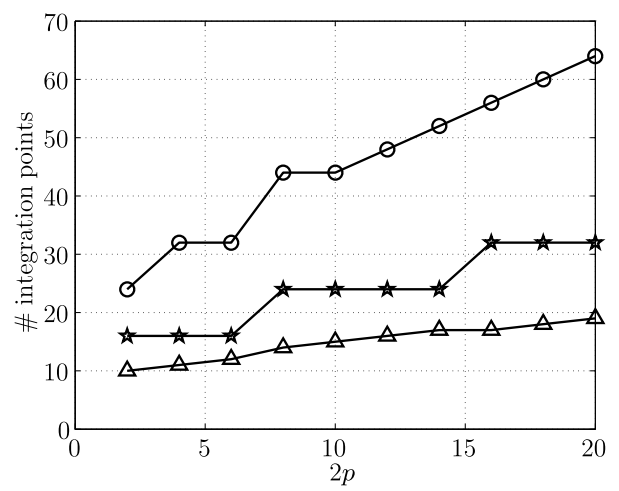

(b) 


\section{Legendre quadratures in each direction as}

$\int_{\Omega_{e}} f d \Omega \simeq \sum_{i=1}^{\mathrm{n}_{\mathrm{ip}}} \sum_{j=1}^{\mathrm{m}_{\mathrm{ip}}} f\left(\boldsymbol{\xi}_{i j}\right)\left|J_{\psi}\left(\lambda_{i}, \vartheta_{j}\right)\right| \omega_{i} \varpi_{j}$,

where $\mathrm{n}_{i p}$ and $\mathrm{m}_{i p}$ are the number of integration points in $\lambda$ and $\vartheta$ directions, respectively, $\boldsymbol{\xi}_{i j}:=\boldsymbol{\psi}\left(\lambda_{i}, \vartheta_{j}\right)$, and $\left\{\lambda_{i}, \omega_{i}\right\}_{i=1}^{\mathrm{n}_{\mathrm{ip}}}$ and $\left\{\vartheta_{j}, \varpi_{j}\right\}_{j=1}^{\mathrm{m}_{\mathrm{ip}}}$ are the 1D quadrature points and weights for $\left[\lambda_{1}^{e}, \lambda_{2}^{e}\right]$ and $[0,1]$ respectively.

Remark 1 When the transformation from the rectangle $\psi$ is considered, the integrals involved in the elemental matrices, for a NEFEM solution with interpolation of degree $p$, can be exactly computed for one of the parameters, $\vartheta$, using a Gauss-Legendre quadrature with $p+1$ integration points. The other dimension, $\lambda$, can be integrated using the same quadrature considered for line integrals over NURBS.

Note that the rational definition of application $\psi$ is only due to the rational definition of the boundary. Thus, in the particular case of a geometry given by a $q$-th degree B-spline, i.e. a piecewise polynomial parametrization, the elemental matrices can be exactly computed with GaussLegendre quadratures with $p+1$ integration points for the $\vartheta$ parameter, and $q(p+1)$ integration points for the NURBS parameter $\lambda$.

\subsubsection{D Case}

Curved faces on a NEFEM tetrahedral mesh can be classified in boundary faces or curved faces with at least one edge on a NURBS boundary. To reduce casuistics in the implementation (i.e., to avoid implementing a different parametrization for each curved face), faces with several edges on different NURBS boundaries are split in subfaces with only one edge on a NURBS boundary. It is worth remarking that subdivisions are only applied to design a numerical quadrature without a special treatment of each face typology, no new degrees of freedom are introduced.

To illustrate the proposed strategy, let us consider a face with two edges on different NURBS boundaries, see Fig. 23. Curved face $\Upsilon_{e}^{E}$ is split in three subfaces, which are defined as a linear convex combination of the edges of $\Upsilon_{e}^{E}$ and its center of mass $\boldsymbol{x}_{C}^{F}$, see Fig. 23. After subdivision each subface has at most one edge on a NURBS boundary. In the example of Fig. 23, two subfaces have one edge on a NURBS boundary (they are parametrized using application $\Theta$ in (5)), and the third face, given by $\boldsymbol{x}_{2}, \boldsymbol{x}_{1}$ and $\boldsymbol{x}_{C}^{F}$, is planar.

With this splitting technique, it is only necessary to describe the strategy to perform the numerical integration on curved boundary faces and curved faces with only one edge on a NURBS boundary.

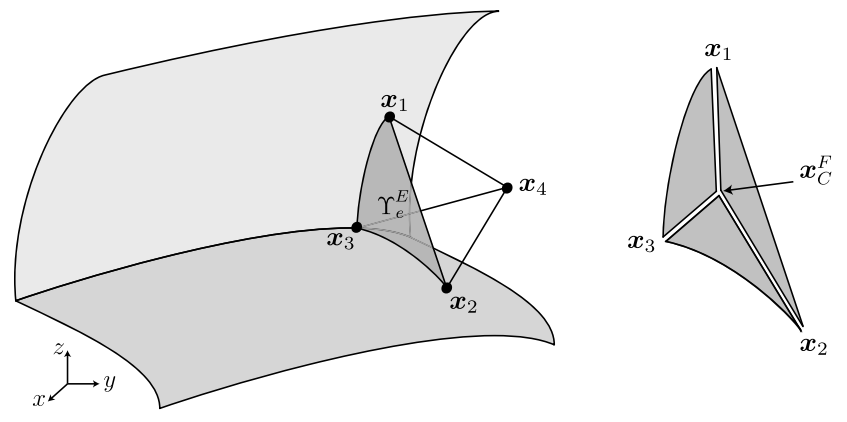

Fig. 23 Tetrahedral element with two edges on different NURBS boundaries, and face splitting used for numerical integration (no new degrees of freedom are introduced)

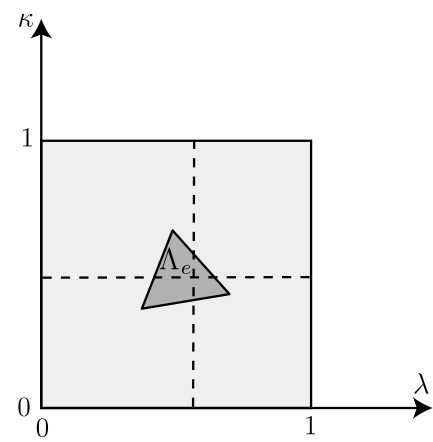

(a)

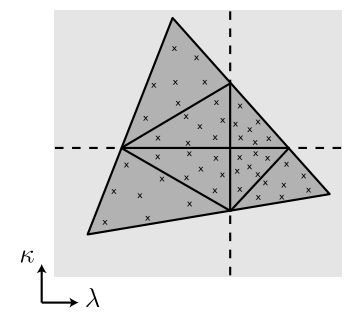

(b)
Fig. 24 Definition of a numerical quadrature on $\Lambda_{e}$ for the numerical integration on a curved tetrahedral face with changes of NURBS parametrization along discontinuous lines (knot lines): (a) triangle in the parametric space and (b) detailed view of the composite quadrature

A surface integral on a curved boundary face $\Upsilon_{e}=$ $S\left(\Lambda_{e}\right)$, see Fig. 11, can be written as

$\int_{\Upsilon_{e}} f d A=\int_{\Lambda_{e}} f(S(\lambda, \kappa))\left\|J_{S}(\lambda, \kappa)\right\| d A$,

where $f$ is a generic function (here a polynomial) and $\left\|J_{S}(\lambda, \kappa)\right\|$ denotes the norm of the differential of the NURBS parametrization $S$ (which, in general, is not a polynomial). An efficient option to evaluate integral (12) is to use a triangle quadrature $[65,85,97]$ in $\Lambda_{e}$. Recall that the spatial discretization is independent on the NURBS boundary representation. Therefore, a boundary face can be intersected by knot lines of the NURBS surface, see Fig. 9. If changes of NURBS parametrization are present within the parametric triangle $\Lambda_{e}$, a numerical quadrature must be designed to account for the piecewise NURBS parametrization. For instance, a triangulation of $\Lambda_{e}$ such that each $s u b$ triangle has no changes of NURBS parametrization can be considered, with the associated composite quadrature (triangle quadrature in each subtriangle), see Fig. 24. 
An integral on a curved face $\Upsilon_{e}^{E}$ with an edge on the NURBS boundary, see Fig. 11, can be written as

$$
\int_{\Upsilon_{e}^{E}} f d A=\int_{\varrho_{1}}^{\varrho_{2}} \int_{0}^{1} f(\boldsymbol{\Theta}(\varrho, \sigma))\left\|J_{\Theta}(\varrho, \sigma)\right\| d A,
$$

where $f$ is a generic function and $\left\|J_{\Theta}(\varrho, \sigma)\right\|$ denotes the norm of the differential of mapping $\boldsymbol{\Theta}$, defined in (5), which in general is not a polynomial. Numerical integration can be performed using 1D Gauss-Legendre quadratures in each direction. In fact, application $\boldsymbol{\Theta}$ is linear in the second parameter, $\sigma$, and exact integration is feasible in this direction. For a NEFEM solution with a degree of approximation $p$, integral in (13) can be exactly computed for this direction, using a Gauss-Legendre quadrature with $p+1$ integration points. Numerical integration for the first direction, given by NURBS parameter $\varrho$, presents the same difficulty as integration over a NURBS curve.

As usual, the evaluation of integral in (13) requires taking into account the piecewise nature of the NURBS parametrization, considering composite quadratures for $\varrho$ direction.

The strategy to perform the element integrals follows the same rationale. Elements with several faces and/or edges on

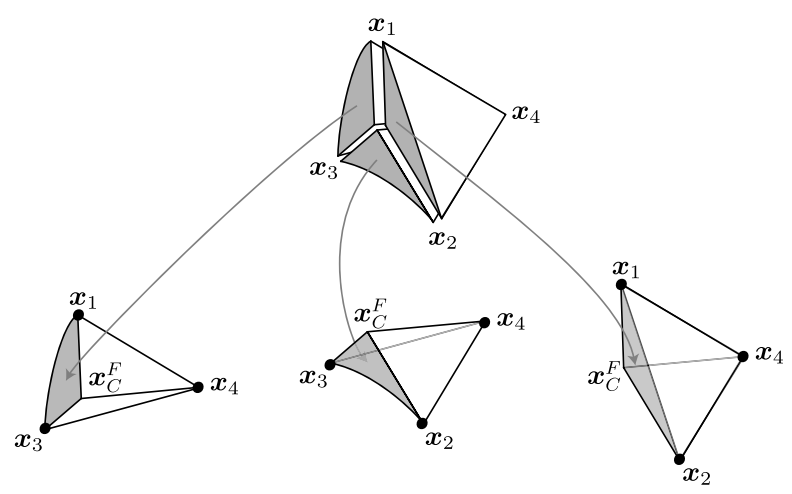

Fig. 25 Splitting used for numerical integration in an element with two edges defined by different NURBS (no new degrees of freedom are introduced) different NURBS boundaries are split in subelements with only one face or one edge on a NURBS boundary. Again, it is worth remarking that subdivisions are only applied to design a numerical quadrature without a special treatment of each element typology, no new degrees of freedom are introduced.

Two examples are presented to illustrate the proposed strategy. First example considers a tetrahedral element with two edges on different NURBS boundaries, see Fig. 23. To design a numerical quadrature on $\Omega_{e}$, three subelements are defined as a linear convex combination of the subfaces and interior vertex of the element, $\boldsymbol{x}_{4}$, see Fig. 25. In this example, two subelements have one edge on a NURBS boundary and the third one has planar faces. Second example considers an element $\Omega_{e}$ with two faces on different NURBS boundaries, as represented in Fig. 26. To perform numerical integration in $\Omega_{e}$, the element is split in four subelements using its center of mass, $\boldsymbol{x}_{C}^{E}$. Subelements are defined as a linear convex combination of $\boldsymbol{x}_{C}^{E}$ and original faces of $\Omega_{e}$, having at most one face on a NURBS boundary.

By combination of these two subdivision strategies, any element with several faces and/or edges on the NURBS boundary can be split into elements with only one face or one edge on the NURBS boundary. Thus, it is only necessary to describe the strategy to perform the numerical integration on these two element typologies.

Volume integrals for an element with one face on a NURBS boundary are performed using parametrization in (6) as

$\int_{\Omega_{e}} f d V=\int_{\Lambda_{e}} \int_{0}^{1} f(\boldsymbol{\Psi}(\lambda, \kappa, \vartheta))\left|J_{\Psi}(\lambda, \kappa, \vartheta)\right| d V$,

where $f$ is a generic function (here a polynomial), and $\left|J_{\Psi}\right|$ denotes the determinant of the Jacobian of transformation $\boldsymbol{\Psi}$. A numerical quadrature on $\Lambda_{e} \times[0,1]$ is easily defined as a tensor product of a triangle quadrature in $\Lambda_{e}$ and a 1D Gauss-Legendre quadrature in [0, 1], see Fig. 27. In fact, exact integration is feasible in third parameter due to
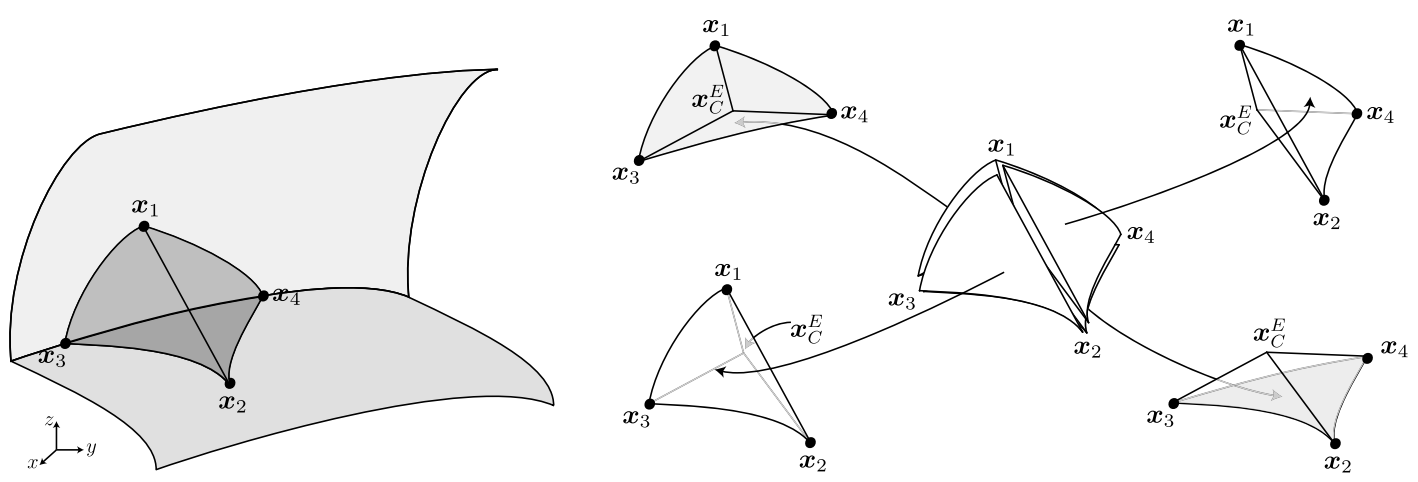

Fig. 26 Splitting used for numerical integration in an element with two faces defined by different NURBS (no new degrees of freedom are introduced) 


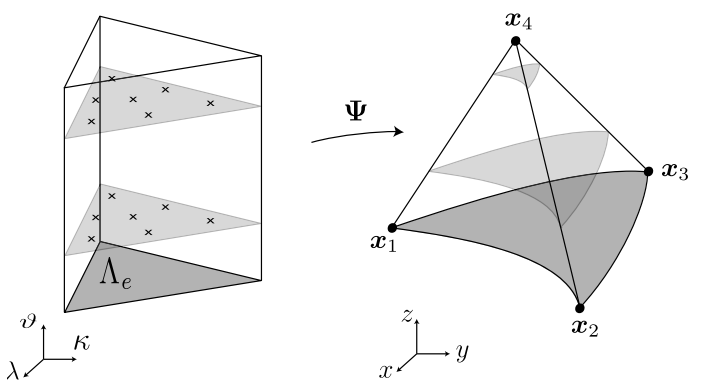

Fig. 27 Transformation from $\Lambda_{e} \times[0,1]$ to $\Omega_{e}$ to perform numerical integration on an element with a face on the NURBS boundary

the linearity of $\boldsymbol{\Psi}$ with respect to $\vartheta$. For a NEFEM solution with a degree of approximation $p$, exact integration in this direction is provided by a Gauss-Legendre quadrature with $p+2$ integration points. To account for changes of NURBS parametrization, only the quadrature in $\Lambda_{e}$ is modified, see Fig. 24.

Similarly, for an element with one edge on a NURBS boundary, volume integrals are performed using parametrization in (7) as

$\int_{\Omega_{e}} f d V=\int_{\varrho_{1}}^{\varrho_{2}} \int_{0}^{1} \int_{0}^{1} f(\boldsymbol{\Phi}(\varrho, \sigma, \tau))\left|J_{\boldsymbol{\Phi}}(\varrho, \sigma, \tau)\right| d V$,

where $\left|J_{\boldsymbol{\Phi}}\right|$ is the determinant of the Jacobian of transformation $\boldsymbol{\Phi}$. Note that application $\boldsymbol{\Phi}$ is linear in second and third parameters, $\sigma$ and $\tau$. Therefore, integrals involved in the elemental matrices, for a NEFEM solution with interpolation of degree $p$, can be exactly computed for these directions using a Gauss-Legendre quadrature with $p+2$ integration points. No exact integration is feasible in NURBS parameter $\varrho$, and composite quadratures must be considered if changes of NURBS parametrization are present.

\subsection{A Priori Error Estimates}

Since NEFEM considers standard FE polynomial interpolation, see Sect. 4.3, a priori error estimates have similar expressions to those of classical FE. For instance, the result for the solution of second-order elliptic problems is recalled in the following theorem.

Theorem 1 Let $\mathcal{T}_{h}$ be a non-degenerate discretization in elements (i.e. there is a positive constant $\beta$ such that $\varrho_{e} / h_{e} \geq$ $\beta$, for all $\Omega_{e} \in \mathcal{T}_{h}$, where $h_{e}$ and $\varrho_{e}$ are the diameters of $\Omega_{e}$ and of the sphere inscribed in $\Omega_{e}$, respectively). Assuming that all boundary conditions along curved boundaries are imposed in weak form and no interior curved faces/edges are present in the mesh, the following a priori estimate holds

$\left\|u-u_{h}\right\|_{E(\Omega)} \leq K h^{p}|u|_{\mathcal{H}^{p}(\Omega)}$, where $\|\cdot\|_{E(\Omega)}$ is the energy norm, $u \in \mathcal{H}^{p+1}(\Omega)$ and $u_{h}$ are the exact and the NEFEM solutions respectively, $K$ is a constant depending on $\beta, h$ is the mesh size, and $p$ is the polynomial degree of interpolation.

Moreover, for p-refinement convergence the following estimate also holds,

$\left\|u-u_{h}\right\|_{E(\Omega)} \leq C \exp \left(-k N^{r}\right)$,

where $C$ and $k$ are positive constants, $N$ is the number of degrees of freedom, and $r \gtrsim 1 / \mathrm{n}_{\mathrm{sd}}$, with $\mathrm{n}_{\mathrm{sd}}$ the number of spatial dimensions.

With NEFEM (as well as for FE in a domain with polygonal boundaries) the spatial discretization does not introduce geometric errors. Moreover, NEFEM uses polynomials to approximate the solution in Cartesian coordinates, see Sect. 4.3. Consequently, all a priori error estimates demonstrated for FE in domains with polygonal boundaries can be reproduced exactly for NEFEM, even in the presence of elements far from having straight edges. The results for standard FE in domains with polygonal boundaries can be found in $[13,52,73]$ for $h$-refinement and in $[3,41,86]$ for $p$ refinement.

It is worth noting that contrary to NEFEM, the proof of a priori error estimates for isoparametric $\mathrm{FE}$ in the presence of curved boundaries requires special attention. This issue is discussed in detail in Sect. 5.3.

Theorem 1 assumes that essential boundary conditions are imposed in weak form, for instance with numerical fluxes in a DG context, or with Nitsche's method in a continuous formulation, see for instance $[36,70]$. If Dirichlet boundary conditions are imposed in strong form, an additional condition is required to keep optimal convergence rates: optimal nodal distributions on every curved element have to be considered, see Fig. 19(b). Theorem 1 also assumes that no interior curved faces/edges are present in the mesh. Again, this extra hypothesis is only needed if a continuous Galerkin approximation is considered and optimal nodal distributions are required to keep optimal convergence rates. This is formally stated in the next result.

Theorem 2 Under the assumptions of Theorem 1, the error bounds (14) and (15) hold for NEFEM in a continuous Galerkin framework, if optimal nodal distributions on every curved element along the Dirichlet boundary or with interior curved faces/edges are considered.

The requirement of Fekette nodal distributions is necessary for an accurate interpolation of Dirichlet boundary conditions on curved boundaries. Due to the use of polynomial nodal basis in Cartesian coordinates, the errors in the approximation of the prescribed value along the boundary may deteriorate the convergence of the solution. This is the 
case for NEFEM as well as for other approaches considering Cartesian polynomial approximations. For instance, in [78] optimal convergence rates are proven when nodes on the boundary correspond to Lobatto points (i.e. Fekette points in 1D).

\subsection{Implementation}

Introducing the NEFEM concept into an existing FE code requires little effort. Note that the main difference of a NEFEM code with a standard FE code is at the level of the computation of elemental matrices and vectors for curved elements and faces (3D) or edges (2D). In fact, fortunately, the usual routines for the computation of elemental matrices and vectors for straight-sided elements can be directly used, without any modification. The usual input of these routines are the integration points and the shape functions evaluated at these points. In the case of curved elements intersecting the NURBS boundary, these inputs are computed as described in previous sections. Thus, most of the routines usual in a standard FE code (routines for assembly, computation of elemental matrices and vectors, etc.) can be directly used.

The most crucial point in the implementation may be the inclusion of the NURBS boundary information. The information of all NURBS describing the boundary and the associated NURBS information for each curved element have to be stored.

In 2D, for every NURBS boundary the associated knot vector and the control polygon are necessary. For each curved edge on a NURBS boundary $\Gamma^{e}$, the parametric coordinates of the boundary nodes $\lambda_{1}^{e}$ and $\lambda_{2}^{e}$, and a pointer to the corresponding NURBS curve $\boldsymbol{C}$ has to be stored, see Fig. 10. In order to compute the parameters $\lambda_{1}^{e}$ and $\lambda_{2}^{e}$, a NURBS projection algorithm can be easily implemented, see for instance $[66,72]$. It is worth noting that the efficiency of the projection algorithm is not crucial because it is performed once at the preprocessing stage.

In 3D, for every NURBS boundary the associated knot vectors and the control net are necessary. For each curved face on the NURBS boundary $\Upsilon_{e}$, the parametric coordinates of the boundary nodes $\left(\lambda_{1}^{e}, \kappa_{1}^{e}\right),\left(\lambda_{2}^{e}, \kappa_{2}^{e}\right)$ and $\left(\lambda_{3}^{e}, \kappa_{3}^{e}\right)$, and a pointer to the corresponding NURBS surface $S$ are stored, see Fig. 11.

It is worth noting that for trimmed and singular surfaces extra information must be stored. First, for each edge on the NURBS boundary corresponding to a trimming curve, see Fig. 12, the parametric coordinates of the edge vertices associated to the trimming curve and a pointer to that curve has to be stored. Second, for each vertex corresponding to a singular point of a NURBS surface two parametric coordinates must be stored, see Fig. 13.

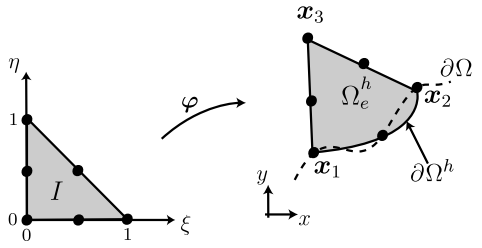

Fig. 28 Isoparametric mapping between the reference element $I$, with local coordinates $\xi$, and an approximation of the physical subdomain in Cartesian coordinates $x$, namely $\Omega_{e}^{h}=\varphi(I)$

Obviously, routines for the evaluation of NURBS and their derivatives at a given parametric coordinates are necessary. This routines can be easily obtained or implemented, see [72] and reference therein.

\section{Why NEFEM?}

This section is devoted to recall and compare several methodologies for the treatment of curved boundaries: isoparametric FEM and Cartesian FEM, with an approximate description of the geometry, and $p$-FEM with an exact boundary representation. To simplify the presentation, triangular elements with one curved side are considered.

Let $\Omega \subset \mathbb{R}^{2}$ be an open bounded domain whose boundary $\partial \Omega$, or a portion of it, is curved. A regular partition of the domain $\bar{\Omega}=\bigcup_{e} \bar{\Omega}_{e}$ in triangular elements is assumed, such that $\Omega_{i} \cap \Omega_{j}=\emptyset$, for $i \neq j$. It is important to remark that, in the following, $\Omega_{e}$ denotes the element with an exact description of the curved boundary, also referred as physical subdomain. This is not the case of classical isoparametric FE, where the computational element, $\Omega_{e}^{h}$, corresponds to a polynomial approximation of the curved boundary.

\subsection{Approximated Boundary Representation}

The standard FE technique used in the presence of curved boundaries is isoparametric FEM, see [35, 104]. A nodal interpolation of the solution, $u$, is considered in the reference element $I$ with local coordinates $\xi=(\xi, \eta)$, see Fig. 28,

$u(\xi) \simeq u^{h}(\xi)=\sum_{i=1}^{\mathrm{n}_{\mathrm{en}}} u_{i} N_{i}(\xi)$,

where $u_{i}$ are nodal values, $N_{i}$ are polynomial shape functions of order $p$ in $\boldsymbol{\xi}$, and $\mathrm{n}_{\mathrm{en}}$ is the number of element nodes. The isoparametric transformation is used to relate local and Cartesian coordinates

$$
\begin{aligned}
\boldsymbol{\varphi}: I \longrightarrow \Omega_{e}^{h} & \\
\boldsymbol{\xi} & \longmapsto \varphi(\xi):=\sum_{i=1}^{\mathrm{n}_{\mathrm{en}}} \boldsymbol{x}_{i} N_{i}(\boldsymbol{\xi}),
\end{aligned}
$$


where $\boldsymbol{x}_{i}$ are the nodal coordinates of the computational element $\Omega_{e}^{h}$. Note that $\Omega_{e}^{h}$ is a polynomial approximation of the physical subdomain $\Omega_{e}$, in particular, of its boundary, see Fig. 28. In fact, the term isoparametric stands for the use of the same polynomial shape functions to define the functional approximation $u^{h}$, and to describe the geometry of the computational element in Cartesian coordinates, see (17).

Numerical integration in the computational element $\Omega_{e}^{h}$ (approximation of $\Omega_{e}$ ) is performed using the isoparametric transformation given in (17), with a numerical quadrature in the reference element $I$. For instance, a stiffness elemental matrix coefficient is computed as

$$
\begin{aligned}
K_{i j}^{e} & =\int_{\Omega_{e}^{h}} \nabla_{x} N_{i}(\xi(\boldsymbol{x})) \cdot \nabla_{x} N_{i}(\xi(\boldsymbol{x})) d \Omega \\
& =\int_{I}\left(\boldsymbol{J}_{\varphi}^{-1} \nabla_{\xi} N_{i}(\xi)\right) \cdot\left(\boldsymbol{J}_{\varphi}^{-1} \nabla_{\xi} N_{j}(\xi)\right)\left|\boldsymbol{J}_{\varphi}\right| d \xi,
\end{aligned}
$$

where $\boldsymbol{J}_{\varphi}$ is the Jacobian of the isoparametric transformation, see (17). For curved elements the isoparametric mapping is non-linear. Therefore, the inverse of the Jacobian, $J_{\varphi}^{-1}$, is not a polynomial function, and no exact integration is feasible with standard quadrature rules. In practice, a symmetric triangle quadrature [97] on $I$, with a sufficiently large number of integration points, is usually employed to compute (18). In fact, a quadrature of order $2 p-1$ provides optimal convergence of the isoparametric FEM, see [106].

There are two sources of error in isoparametric FEM. First, the isoparametric mapping in (17) introduces geometric errors, due to the approximation of the physical subdomain $\Omega_{e}$ by the computational element $\Omega_{e}^{h}$. In fact, the boundary of the computational domain $\partial \Omega_{h}$ is a piecewise polynomial approximation of the exact boundary $\partial \Omega$, see Fig. 28. Second, for high-order approximations on curved elements, the definition of the polynomial interpolation in (16) in local coordinates, $\xi$, implies a loss of consistency: a polynomial interpolation of degree $p>1$ in $\xi$ does not correspond to a polynomial interpolation of degree $p$ in $\boldsymbol{x}$. This implies that the approximation is able to reproduce linear functions but it is not able to reproduce higher order polynomials in Cartesian coordinates. In other words, curved isoparametric FEs pass the patch test but they fail to pass the so-called higher order patch tests, see [106] for further details.

Remark 2 Optimal convergence of isoparametric FEs is obtained under some smoothness assumptions on the isoparametric mapping. In practice, a specific node placement of interior nodes in curved elements of order $p>2$ is mandatory to guarantee optimal rates of $h$ and $p$ convergence, see $[17,61]$.
An alternative to ensure consistency of the approximation, and optimal convergence for any nodal distribution, is the so-called Cartesian FEM. This approach defines the polynomial basis for the approximation of the solution directly in the physical space, with Cartesian coordinates $\boldsymbol{x}$. Nevertheless, the isoparametric transformation in (28) and the computational element $\Omega_{e}^{h}$, are still considered for integration purposes. For instance, a stiffness elemental matrix coefficient is computed as

$$
\begin{aligned}
K_{i j}^{e} & =\int_{\Omega_{e}^{h}} \nabla_{x} N_{i}(\boldsymbol{x}) \cdot \nabla_{x} N_{j}(\boldsymbol{x}) d \Omega \\
& =\int_{I} \nabla_{\boldsymbol{x}} N_{i}(\boldsymbol{x}(\boldsymbol{\xi})) \cdot \nabla_{\boldsymbol{x}} N_{j}(\boldsymbol{x}(\boldsymbol{\xi}))\left|\boldsymbol{J}_{\boldsymbol{\varphi}}\right| d \boldsymbol{\xi} .
\end{aligned}
$$

The definition of the approximation with Cartesian coordinates, $\boldsymbol{x}$, ensures reproducibility of polynomials (i.e., consistency of order $p$ ). Moreover, exact integration is feasible because shape functions and their derivatives are polynomials, not only with Cartesian coordinates $\boldsymbol{x}$, but also with local coordinates $\boldsymbol{\xi}$. More precisely, for a degree of interpolation $p, N_{i}(\boldsymbol{x}(\boldsymbol{\xi}))$ and $\nabla_{\boldsymbol{x}} N_{i}(\boldsymbol{x}(\boldsymbol{\xi}))$ are polynomials of degree $p$ and $p-1$, respectively. Therefore, the function to be integrated, $f(\boldsymbol{\xi})=\nabla_{\boldsymbol{x}} N_{i}(\boldsymbol{x}(\boldsymbol{\xi})) \cdot \nabla_{\boldsymbol{x}} N_{j}(\boldsymbol{x}(\boldsymbol{\xi}))\left|\boldsymbol{J}_{\varphi}\right|$, is a polynomial of degree $p(4 p-3)$ in $\xi$. The evaluation of integral in (19) can be exact with a triangle quadrature of order $p(4 p-3)$ on the reference element $I$.

It is worth noting that Cartesian approximation can be considered with Lagrangian, Eulerian, arbitrary LagrangianEulerian or updated Lagrangian formulations. Obviously, Cartesian approximation introduces an overhead with respect to isoparametric FEs because it requires a specific definition of the approximation for each curved element. When the mesh is fixed, this overhead is restricted to elements affected by the curved boundary description, usually a very small portion of the total number of elements. For meshes evolving with the simulation this overhead is repeated each time step, and internal curved edges must be considered. It is worth remarking that the extra cost introduced by Cartesian FEM is justified by the improved accuracy with respect to isoparametric FEs. In addition, Cartesian approximation allows to ensure optimal convergence with no dependence on the node placement for curved elements, see Remark 2 and Sect. 5.3.

Although Cartesian FEM ensures reproducibility of polynomials in the physical space, the numerical integration in Cartesian FEM is still done in the (approximated) computational element $\Omega_{e}^{h}$. Thus, Cartesian FEM precludes the lack consistency of isoparametric FEM, but it still suffers from geometric error. This is not the case for $p$-FEM and NEFEM formulations. 


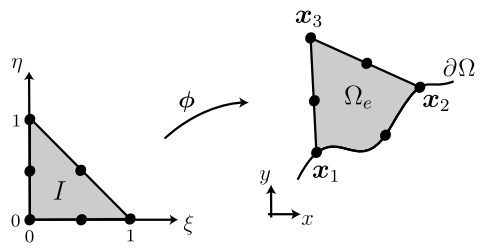

Fig. 29 Exact mapping between the reference element $I$ with local coordinates $\xi$, and the physical subdomain $\Omega_{e}$ with Cartesian coordinates $\boldsymbol{x}$

\subsection{NURBS Boundary Representation}

This section recalls the basics of $p$-FEM formulation considering an exact boundary representation, see [86, 87].

Nodal interpolation in $p$-FEM is defined in the reference element using local coordinates $\xi$, see (16), but an exact mapping is employed between the reference element $I$ and the physical subdomain $\Omega_{e}$. For instance, assuming a NURBS parametrization $C(\xi)$ of the curved edge of $\Omega_{e}$, a simple $p$-FEM mapping may be

$$
\phi: I \longrightarrow \Omega_{e}
$$

$$
\xi \longmapsto \phi(\xi):=\frac{1-\xi-\eta}{1-\xi} \boldsymbol{C}(\xi)+\frac{\xi \eta}{1-\xi} x_{2}+\eta x_{3},
$$

where $\boldsymbol{x}_{1}=\boldsymbol{C}(0)$ and $\boldsymbol{x}_{2}=\boldsymbol{C}(1)$ are the vertices of $\Omega_{e}$ on the curved boundary, and $\boldsymbol{x}_{3}$ is the internal vertex, see Fig. 29. Other options are possible in order to define an exact mapping from $I$ to $\Omega_{e}$, see for instance [71]. However, no relevant differences, depending on the particular mapping $\phi$, are observed in the numerical tests discussed in Sect. 6 .

In $p$-FEM, a stiffness elemental matrix coefficient is computed as

$$
\begin{aligned}
K_{i j}^{e} & =\int_{\Omega_{e}} \nabla_{x} N_{i}(\xi(\boldsymbol{x})) \cdot \nabla_{x} N_{i}(\xi(\boldsymbol{x})) d \Omega \\
& =\int_{I}\left(\boldsymbol{J}_{\boldsymbol{\phi}}^{-1} \nabla_{\xi} N_{i}(\boldsymbol{\xi})\right) \cdot\left(\boldsymbol{J}_{\boldsymbol{\phi}}^{-1} \nabla_{\xi} N_{j}(\boldsymbol{\xi})\right)\left|\boldsymbol{J}_{\boldsymbol{\phi}}\right| d \boldsymbol{\xi},
\end{aligned}
$$

integrating over the physical subdomain $\Omega_{e}$, with an exact description of the geometry. Note that, the inverse of the Jacobian, $\boldsymbol{J}_{\boldsymbol{\phi}}^{-1}$, is not a polynomial function and, as for the isoparametric FEM, no exact integration is feasible with standard quadrature rules. Nevertheless, under some smoothness requirements on the parametrization $C(\xi)$, the same quadrature order used in the isoparametric FEM, that is $2 p-1$, guarantees optimal convergence for $p$-FEM, see [6].

Note that $p$-FEM presents a major advantage, compared to isoparametric or Cartesian FEM, which is the exact boundary representation. Nevertheless, $p$-FEM still suffers the same lack of consistency as isoparametric FEM, due to the definition of the polynomial shape functions in the reference element $I$, with local coordinates $\xi$. This is not the case for NEFEM, see Sect. 4.3.
Fig. 30 Triangle with a curved

edge containing changes of NURBS parametrization (marked with $\square$ )

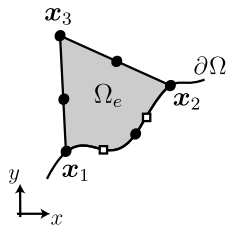

NEFEM considers the polynomial approximation with Cartesian coordinates $\boldsymbol{x}$, ensuring reproducibility of polynomials in the physical space for any order of approximation $p$. The exact description of the boundary is used to perform the numerical integration on the physical subdomain $\Omega_{e}$. In NEFEM, a stiffness elemental matrix coefficient is computed as

$$
\begin{aligned}
K_{i j}^{e} & =\int_{\Omega_{e}} \nabla_{x} N_{i}(\boldsymbol{x}) \cdot \nabla_{x} N_{j}(\boldsymbol{x}) d \Omega \\
& =\int_{R} \nabla_{\boldsymbol{x}} N_{i}(\boldsymbol{x}(\boldsymbol{\lambda})) \cdot \nabla_{\boldsymbol{x}} N_{j}(\boldsymbol{x}(\boldsymbol{\lambda}))\left|\boldsymbol{J}_{\boldsymbol{\psi}}\right| d \boldsymbol{\lambda},
\end{aligned}
$$

using the transformation in (3).

It is worth recalling that NURBS are piecewise rational functions defined in parametric form, see Sect. 3. Therefore, numerical integration for $p$-FEM and NEFEM must be designed to account for changes of NURBS definition along the curved edge of the physical subdomain $\Omega_{e}$. This issue is addressed in the next section.

\subsubsection{Numerical Integration for $p$-FEM and NEFEM}

This section discusses the numerical integration for $p$-FEM and NEFEM when changes of NURBS parametrization are considered inside the curved boundary edge of a physical subdomain $\Omega_{e}$.

For illustration purposes the triangle with a curved edge represented in Fig. 30 is considered first. The curved edge is described with a piecewise parametrization $\boldsymbol{C}$, whose definition changes in two points on the curved edge, marked with $\square$. The parametric coordinates of these points are called the breakpoints or knots of the NURBS parametrization, see Sect. 3.

In $p$-FEM, the piecewise definition of the boundary induces a piecewise definition of the mapping $\boldsymbol{\phi}$, see (20). Therefore, a specifically designed numerical quadrature should be defined in the reference element $I$. For the triangle represented in Fig. 30, with two changes of NURBS definition, the reference element should be partitioned as represented in Fig. 31, where the discontinuous lines show the changes of definition of the mapping $\boldsymbol{\phi}$. Note that these lines originate at the breakpoints of the NURBS parametrization in the $\xi$ axis, and are extended inside the reference element. A composite numerical quadrature on $I$ should be defined by using different numerical quadratures in each region. 
$\partial \Omega_{h}$, is a piecewise polynomial approximation of the exact boundary, $\partial \Omega$. The only method ensuring both consistency of the approximation (for any $p$ ) and an exact boundary representation of the domain is NEFEM, see Table 1.

It is worth mentioning that, from a computational point of view, the definition of the polynomial basis in local coordinates $\xi$, as done in the isoparametric FEM and in $p$-FEM, induces a marginal extra efficiency. In this case the polynomial basis is defined once in the reference element and used to define the approximation in each curved element, whereas a Cartesian approximation requires a specific definition of the polynomial basis for each curved element. The use of the isoparametric transformation to perform the numerical integration, as done in the isoparametric FEM and in the Cartesian FEM, also induces another marginal extra efficiency. A numerical quadrature is defined in the reference element $I$ and used for each curved element. Whereas methods with an exact boundary representation require specific strategies for curved elements. Nevertheless, it is important to recall that this extra cost is restricted to elements affected by the NURBS boundary representation, in most applications a very small portion of the total number of elements, those in contact with non polynomial boundaries.

A priori error estimates for the FE methodologies considered in this work have similar expressions, with optimal convergence in all cases. However, the hypotheses to obtain these estimates are different, depending on the definition of the approximation, in local or Cartesian coordinates, and on the boundary representation, that is, approximated or exact.

The influence of the definition of the polynomial basis in local or Cartesian coordinates is discussed first. When the polynomial basis is defined with local coordinates $\xi$, the mapping relating local and Cartesian coordinates must be smooth enough to guarantee optimal convergence. In practice, for the isoparametric FEM specific nodal distributions on curved elements are necessary to obtain optimal convergence rates with $p>2$, see Remark 2 and [17, 61]. For $p$-FEM, the NURBS parametrization of the curved boundary must be smooth enough to guarantee the necessary smoothness of the $p$-FEM mapping relating local and Cartesian coordinates, see [3]. In contrast, when the polynomial basis is defined with Cartesian coordinates $x$, the derivation of a priori error estimates is very close to FE $a$ priori error estimates in polygonal domains, which can be found in [13, 52]. For Cartesian FEM and NEFEM, no specific nodal distributions in curved elements are necessary to achieve optimal convergence. Moreover, smooth variations of the NURBS parametrization are not required to obtain the optimal convergence rates with NEFEM, see Sect. 4.5. Nevertheless, optimal a priori error estimates for FE methods with a Cartesian approximation require extra attention if a standard (continuous) Galerkin formulation is considered. If a strong imposition of Dirichlet boundary conditions is considered, or if curved internal edges/faces are present in the mesh, optimal nodal distributions in curved elements are necessary in order to guarantee optimal rates of convergence, see Theorems 1 and 2 in Sect. 4.5. The key issue is that test functions do not vanish over the curved boundary, even if the test function is associated to a node that is not located on the boundary. With specific nodal distributions, such as Fekette points, the error induced by this lack of consistency is lower than the approximation error and the optimal convergence is guaranteed. Obviously, this is not the case for weakly imposed Dirichlet boundary conditions, where optimal convergence is obtained irrespectively of the node placement. Recall that in a DG framework boundary conditions are usually imposed in a weak sense, and recent studies also suggest advantages of imposing boundary conditions weakly in a standard continuous Galerkin framework, see [10].

The influence of the boundary representation in the convergence properties of the approximation is discussed next. For FE methods with an approximate boundary representation (isoparametric FEM and Cartesian FEM), optimal convergence is provided under the assumption that geometric errors are lower than the discretization error. The difference between the computational element $\Omega_{e}^{h}$ and the physical subdomain $\Omega_{e}$ must be bounded by $\gamma h^{p}$, where $\gamma$ is a constant, $h$ is the characteristic mesh size and $p$ is the interpolation degree. Moreover, bounds of the Jacobian of the isoparametric transformation and its first $p$ derivatives are also necessary, see [17].

Thus, a curved element with an approximated boundary representation must verify two contradictory requirements. On one hand, the computational (polynomial) boundary $\partial \Omega^{h}$ has to be close enough to the exact boundary $\partial \Omega$. And, on the other hand, the discrepancy between the computational element and the straight-sided element given by its vertices must vanish fast enough, see [61].

\section{Numerical Examples}

In this section the application and performance of NEFEM in the context of continuous and discontinuous Galerkin formulations are illustrated using several $2 \mathrm{D}$ and $3 \mathrm{D}$ examples.

First, the solution of second-order elliptic problems using a standard continuous Galerkin (CG) formulation is presented. A priori error estimates recalled in Sect. 4.5 are verified for both $h$ and $p$ refinement. NEFEM is compared to the methodologies for the treatment of curved boundaries discussed in Sect. 5, namely isoparametric and Cartesian FEM with an approximated boundary representation and $p$-FEM with a NURBS boundary representation. Finally, the issues associated to Cartesian approximations in the context of CG formulations are discussed. 


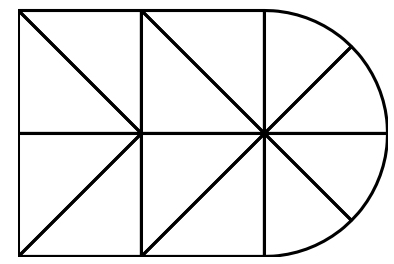

(a) Mesh 1

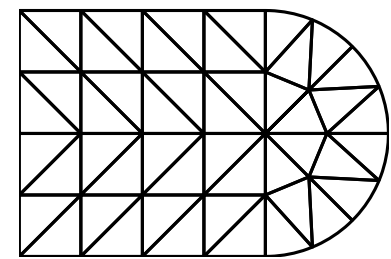

(b) Mesh 2

Fig. 34 Coarse meshes for the second-order elliptic problem. Nested remeshing is used for refinement

Secondly, the solution of inviscid compressible flow problems is considered using a discontinuous Galerkin (DG) formulation. The importance of the geometrical model in this context is explored and the benefits of NEFEM are shown.

Finally, NEFEM is applied to the numerical solution of electromagnetic scattering problems using a DG formulation. The advantages of NEFEM in this context are presented comparing the results to other curved FE techniques and to other methodologies used by the computational electromagnetics (CEM) community. In addition, the possibilities of NEFEM when the size of the geometrical model is subsidiary to the geometric complexity are shown.

\subsection{Second-Order Elliptic Problems}

In this section the following model problem is considered

$$
\begin{cases}-\Delta u+u=f & \text { in } \Omega \\ u=u_{d} & \text { on } \Gamma_{d} \\ \nabla u \cdot \boldsymbol{n}=g_{n} & \text { on } \Gamma_{n}\end{cases}
$$

where $\Omega$ is the domain, $\bar{\Gamma}_{d} \cup \bar{\Gamma}_{n}=\partial \Omega$ and $\boldsymbol{n}$ is the outward unit normal vector on $\partial \Omega$.

First, a $2 \mathrm{D}$ domain $\Omega$ is considered (see two computational meshes with curved elements in Fig. 34). A Dirichlet boundary condition, corresponding to the analytical solution, is imposed in strong form in the polygonal part of the boundary $\Gamma_{d}$, and a Neumann boundary condition, also corresponding to the analytical normal flux, is imposed in the curved part of the boundary $\Gamma_{n}$. If desired, Dirichlet boundary conditions could be imposed in a strong sense over the curved boundary by considering Fekette nodal distributions on curved boundary edges, see Theorem 2 in Sect. 4.5 and the discussion in Sect. 5.3. The curved part of the boundary is given by the usual quadratic NURBS that describes a circle, see [72], trimmed to represent half a circle.

In order to illustrate both the relevance of an accurate boundary representation of the domain and the issue of consistency, a polynomial source term is considered first, such that the analytical solution of the problem is a polynomial function of degree 7 , namely

$u(x, y)=x^{5} y^{2}+x^{3} y^{4}+y^{7}$.

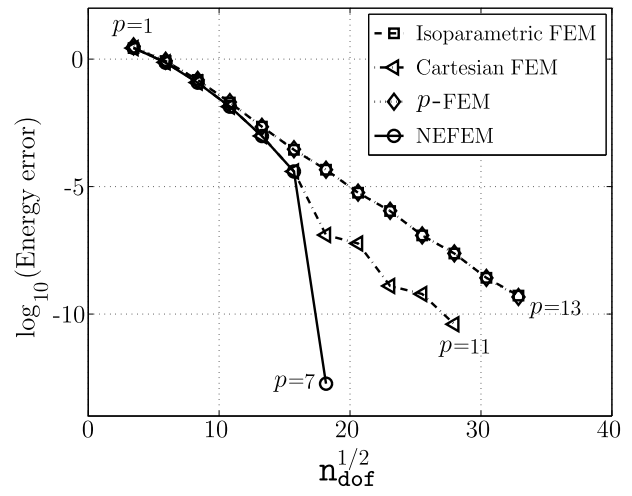

Fig. 35 Poisson problem with polynomial analytical solution: $p$-convergence in the energy norm in the coarse mesh of Fig. 34

Figure 35 shows a $p$-convergence comparison in the coarse mesh of Fig. 34. The energy error is represented as a function of the square root of the number of degrees of freedom $\left(n_{\mathrm{dof}}\right)$ when the polynomial order of the approximation is uniformly increased, starting with $p=1$. In NEFEM, the boundary of the domain is exactly represented and the polynomial basis for the approximation is defined in Cartesian coordinates. Therefore, with a polynomial approximation of degree $p=7$ the solution provided by NEFEM is the exact solution (except from rounding errors). With Cartesian FEs, the basis is also defined in Cartesian coordinates, but the computational boundary is a piecewise polynomial approximation of the circle. Thus, the difference between NEFEM and Cartesian FEM is only due to geometric errors. Although isoparametric FE and the $p$-FEM show the expected (exponential) convergence, the effect of a nonconsistent approximation is clearly observed. The function to be approximated is a polynomial in Cartesian coordinates $u(\boldsymbol{x})$, but it is far from being a polynomial function in local coordinates $u(\xi(\boldsymbol{x}))$. In this example, errors introduced by a non-consistent approximation are higher than geometric errors. Thus, isoparametric FEM and $p$-FEM provide the same performance.

Next, the same second-order elliptic problem is solved with a non-polynomial source term, such that the analytical solution of the problem is

$u(x, y)=x \cos (y)+y \sin (x)$.

Convergence under $h$-refinement is first explored. Figure 34 shows the first two computational meshes; nested remeshing is used for refinement. The number of integration points is sufficiently large in order to ensure that no errors due to numerical integration are present. Energy error is depicted in Fig. 36 for a polynomial approximation of degree $p=5$ and $p=6$. The optimal rate of $h$-convergence is exhibited by every FE technique considered, but some differences in accuracy are observed. In this example the use of a 
Fig. 51 Inviscid subsonic flow around a circle: (a) Pressure loss distribution and (b) pressure coefficient distribution, at the upper mid of the circle for $p=1$

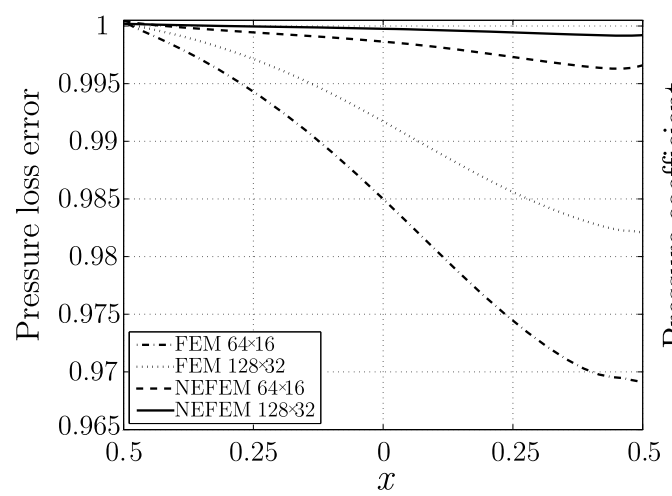

(a)

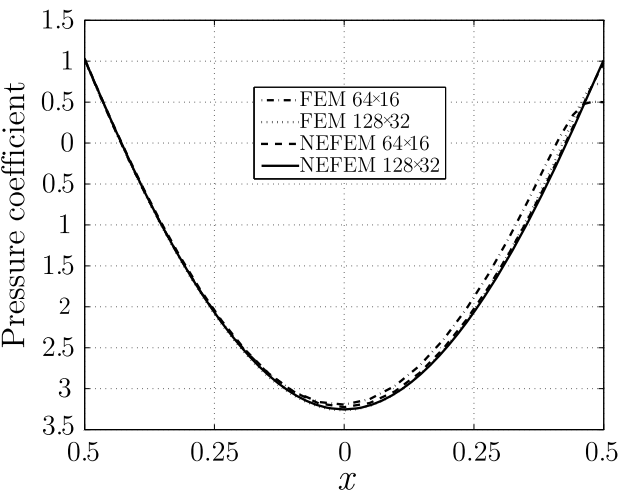

(b)

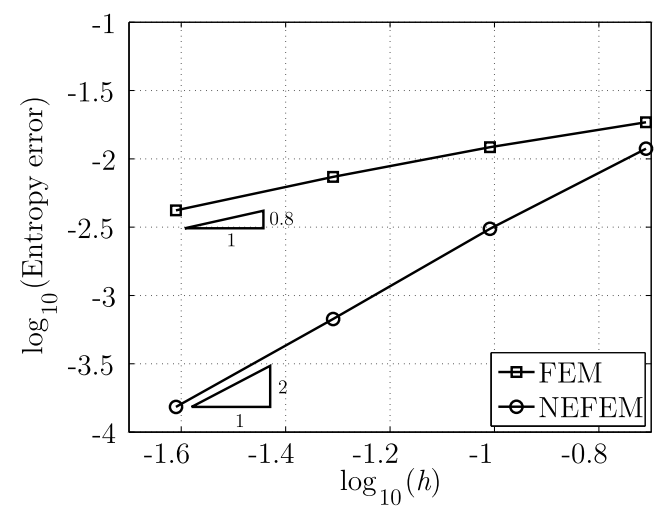

Fig. 52 Inviscid subsonic flow around a circle: $h$-convergence of the entropy error for isoparametric FEs and NEFEM $(p=1)$

very good symmetry of the Mach number patterns, even if coarse meshes are used. More important, NEFEM allows convergence to the correct physical solution using the fine mesh with a piecewise linear approximation of the solution. The exact computation of the outward unit normal improves the imposition of the solid wall boundary condition. This issue and the exact representation of the boundary drastically reduce the entropy production compared to isoparametric FE.

In order to provide a more quantitative analysis, other aerodynamic quantities for the evaluation of the accuracy are used, namely the entropy error

$\epsilon_{\mathrm{ent}}=\frac{\mathrm{p}}{\mathrm{p}_{\infty}}\left(\frac{\rho_{\infty}}{\rho}\right)^{\gamma}-1$

the pressure loss

$\mathrm{p}_{\text {loss }}=\frac{\mathrm{p}}{\mathrm{p}_{\infty}}\left(\frac{1+0.5(\gamma-1) M^{2}}{1+0.5(\gamma-1) M_{\infty}^{2}}\right)^{\frac{\gamma}{\gamma-1}}$

and the pressure coefficient

$C_{\mathrm{p}}=\frac{\mathrm{p}-\mathrm{p}_{\infty}}{0.5 \rho_{\infty} v_{\infty}^{2}}$ where the subscript $\infty$ indicates free-stream values.

Figure 51 shows pressure loss and pressure coefficient distributions on the upper mid of the circle. At the most critical point, the stagnation point behind the circle, the maximum pressure loss error with isoparametric FE in the fine mesh is $1.8 \times 10^{-2}$, whereas NEFEM maximum error is reduced more than one order of magnitude, namely $8.4 \times 10^{-4}$. Moreover, in the fine mesh, the pressure coefficient error at the stagnation point is $2.8 \times 10^{-1}$ for standard FE and $4 \times 10^{-3}$ for NEFEM, almost two orders of magnitude more precise for the same number of degrees of freedom.

Figure 52 represents $h$-convergence of the entropy error on the upper part of the circle. Entropy production observable in Fig. 49 deteriorates the $h$-convergence of standard isoparametric FEs. In contrast, NEFEM exhibits the optimal convergence rate for linear approximation.

The next example involves the subsonic flow over a NACA0012 airfoil at free stream Mach number $M_{\infty}=0.3$ and angle of attack $\alpha=0^{\circ}$. Four O-meshes with $16 \times 4$, $32 \times 8,64 \times 16$, and $128 \times 32$ nodes (i.e. $128,512,2048$ and 8192 elements respectively) are considered for low-order computations. A detailed view of these meshes near the airfoil is represented in Fig. 53. To design such meshes, a conformal mapping is applied to previous meshes used for the flow around a circle.

Figure 54 shows Mach number isolines for isoparametric FE with linear approximation. Again, the results illustrate the spurious entropy production caused by the polygonal approximation of curved boundaries. Figure 55 shows Mach number isolines computed with NEFEM using linear approximation. The results show, once more, a remarkable improvement due to the exact boundary representation. To quantify accuracy, Fig. 56 compare the entropy production and pressure loss in the upper part of the airfoil for isoparametric FE and NEFEM in the finest mesh if Fig. 53. With isoparametric FE the $\mathcal{L}^{2}$ norm of the entropy error on the airfoil profile is $1.3 \times 10^{-3}$ in the finest mesh. The exact boundary representation considered in NEFEM reduces the $\mathcal{L}^{2}$ 
Fig. 60 Inviscid subsonic flow around a circle: coarse mesh and Fekkete nodal distribution for $p=6$

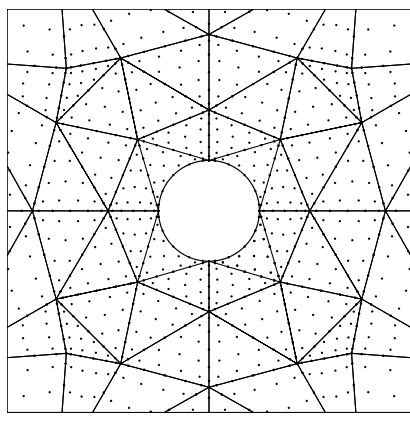

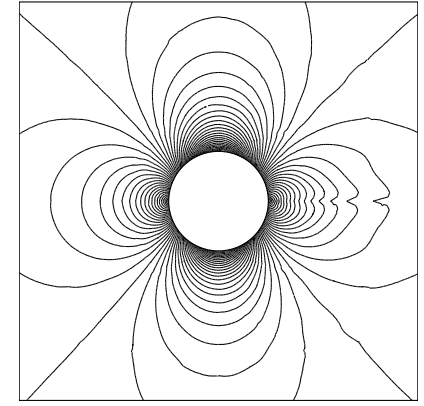

(a) FEM $p=6$

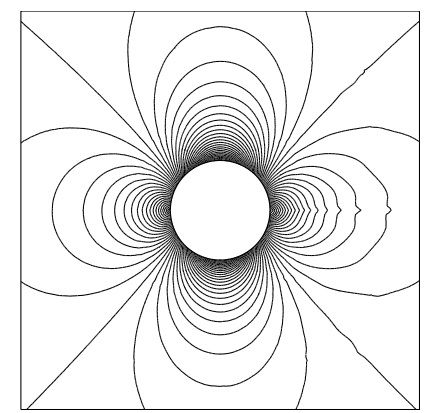

(c) $\operatorname{FEM} p=7$

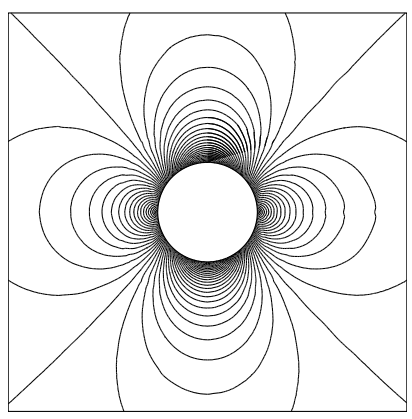

(b) NEFEM $p=6$

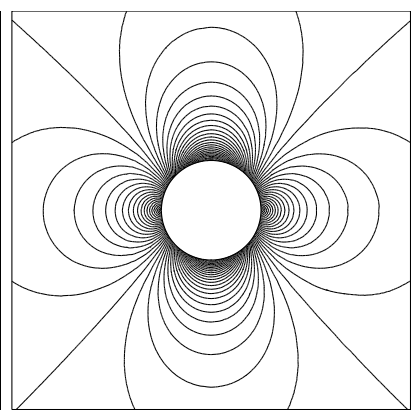

(d) NEFEM $p=7$
Fig. 61 Inviscid subsonic flow around a circle: Mach number distribution in the coarse mesh shown in Fig. 60 with isoparametric FE and NEFEM and high-order approximations

In order to show the benefits of NEFEM, the coarse mesh represented in Fig. 60 is considered and high-order isoparametric FE and NEFEM are compared. It is worth emphasizing that only four curved elements are considered to describe the circle, corresponding to 24 nodes over the curved boundary for a degree of approximation $p=6$. Figure 61 shows the Mach number isolines for isoparametric FEs and NEFEM with a degree of approximation $p=6$ and $p=7$. The solution with very high-order isoparametric FEs is not completely symmetric with respect to the $y$ axis, reflecting a small entropy production behind the circle. It is worth remarking that for $p=6$ the maximum difference between the approximated boundary and the true circle is less than $10^{-4}$ and the asymmetry in the Mach number distribution is remarkable. For $p=7$ the maximum difference between the approximated boundary and the true circle is almost $10^{-5}$ and a visually symmetric Mach number distribution is still

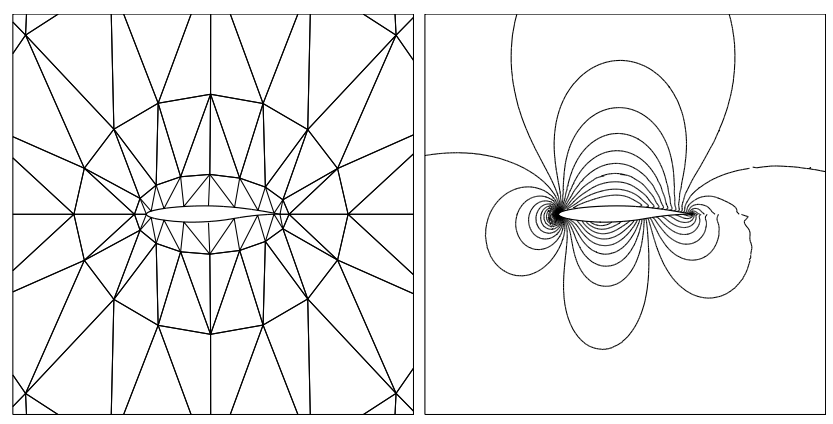

(a) Mesh

(b) Mach number

Fig. 62 Inviscid subsonic flow around a RAE2822 airfoil: coarse mesh and Mach number isolines for NEFEM and $p=8$

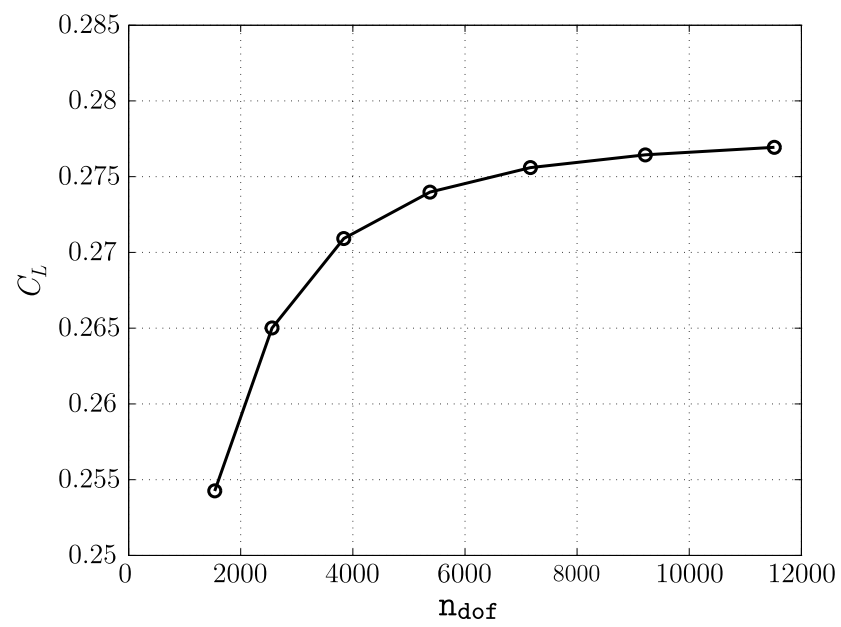

Fig. 63 Inviscid subsonic flow around a RAE2822 airfoil: lift coefficient convergence for increasing $p$ in the mesh of Fig. 62(a)

not obtained. The discontinuity in the outward unite normal in the stagnation point behind the circle, due to the piecewise $\mathcal{C}^{0}$ approximation of the circle with isoparametric FEs, has a big impact in the entropy production, even with very highorder isoparametric approximations. In contrast, it is worth remarking the quality of the solution obtained with NEFEM with a degree of approximation $p=6$. The solution is perfectly symmetric with respect to the $y$ axis showing that the inaccuracies observed with isoparametric FEs are not due to the coarse mesh considered in this example, but to geometric inaccuracies.

The last example in this section involves the simulation of the flow around a (non-symmetric) RAE2822 airfoil at free-stream Mach number $M_{\infty}=0.5$ and angle of attack $\alpha=0^{\circ}$. Figure 62 shows the computational mesh and the Mach number distribution for $p=8$. Despite the ultra coarse mesh considered, with NEFEM and high-order approximations the complex flow features are well resolved. To quantify the accuracy of high-order NEFEM computations, Fig. 63 shows the convergence of the lift coefficient $\left(C_{L}\right)$ as the degree of the approximation is uniformly in- 
Fig. 67 Scattering by a PEC NACA0012 airfoil of chord length $2 \lambda$

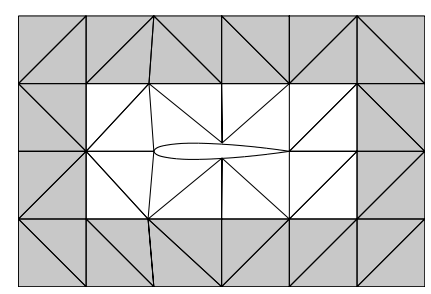

(a) Mesh

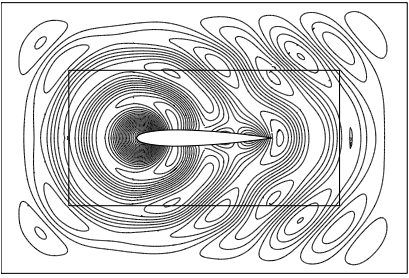

(b) $H_{3}$ field (c) $E_{3}^{T}$ field
Fig. 68 Scattering by a PEC NACA0012 airfoil of chord length $2 \lambda$ : TE RCS comparison as $p$ increases

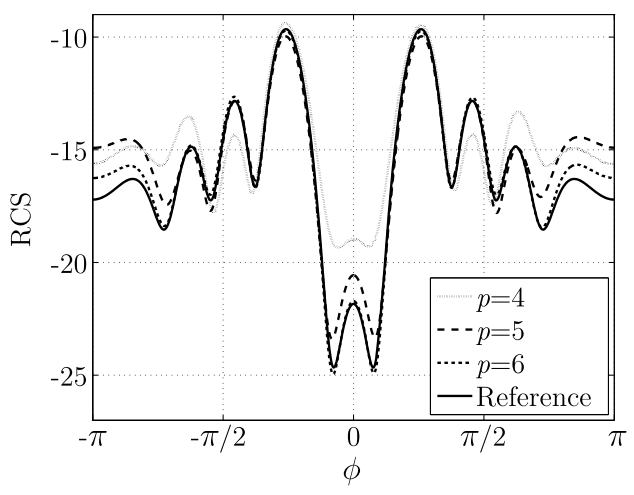

(a) FEM

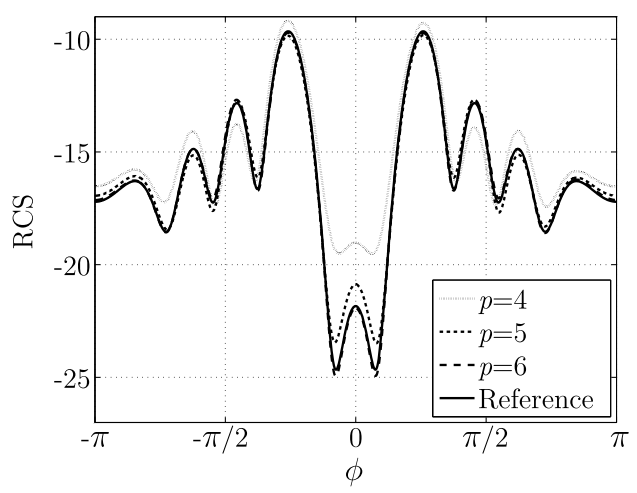

(b) NEFEM

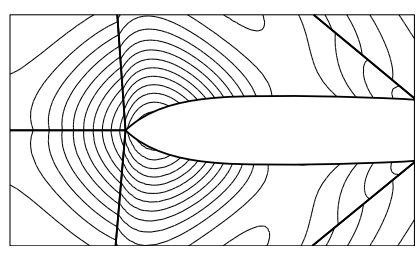

(a) $\operatorname{FEM} p=4$

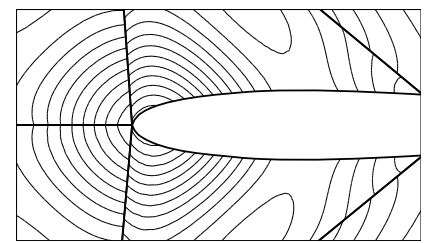

(d) NEFEM $p=4$

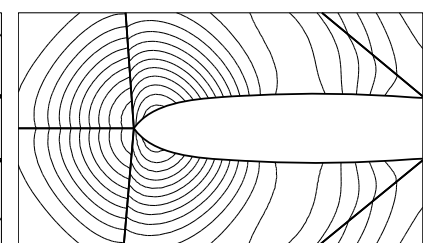

(b) $\operatorname{FEM} p=5$

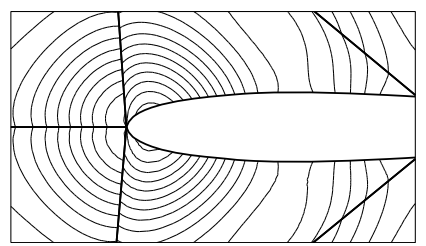

(e) NEFEM $p=5$

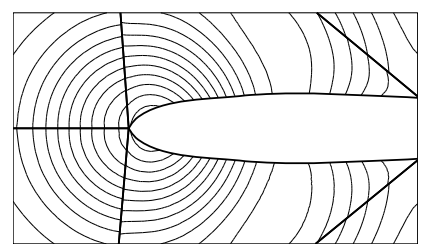

(c) FEM $p=6$

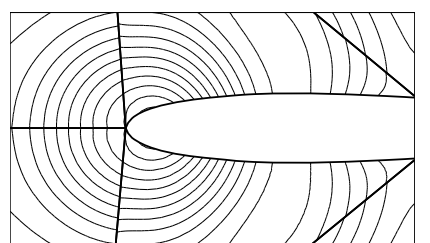

(f) NEFEM $p=6$ length $2 \lambda$ is considered. Figure 67 shows an ultra coarse computational mesh with a $\lambda$ thick PML, the scattered $H_{3}$ field and the total (scattered plus incident) $E_{3}$ field for a NEFEM solution with $p=6$. Note that only two elements are considered in the upper part of the airfoil. Figure 68 illustrates the convergence of the solution for increasing $p$, with isoparametric FEs and NEFEM. As no analytical solution is available, a reference solution is computed in a fine mesh with high-order approximation and a thicker PML.

For isoparametric FEs, the RCS error in $\mathcal{L}^{2}([-\pi, \pi])$ norm decreases as $p$ increases, but it is important to remark that the RCS error in the $\mathcal{L}^{\infty}([-\pi, \pi])$ norm behaves different. In particular, the RCS error for angles near $-\pi$ or $\pi$ is higher with $p=5$ than using $p=4$, showing important dis- crepancies with respect to the reference solution. Recall that, for isoparametric FEs, as $p$ increases not only the solution is represented with higher degree, but also the geometry. Thus, slightly different profiles are considered for each $p$. Moreover, the approximated boundary is only $\mathcal{C}^{0}$ on the boundary nodes. In particular a discontinuity of the profile in the leading edge is clearly observable using coarse meshes and highorder isoparametric FEs, see Figs. 69(a), (b) and (c). With NEFEM, the exact boundary representation is considered with no dependence on the spatial discretization (i.e. the degree of the polynomial approximation), see Figs. 69(d), (e) and (f). Consequently, with NEFEM, the RCS error is uniformly reduced for all viewing angles as the degree of the approximation is increased, see Fig. 68(b). 
Fig. 70 Scattering by a PEC

NACA0012 airfoil of chord length $2 \lambda$ : RCS error comparison for isoparametric FEM and NEFEM

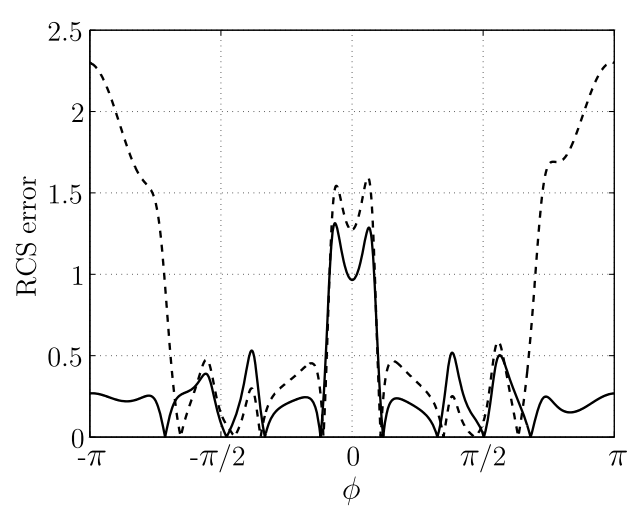

(a) $p=5$

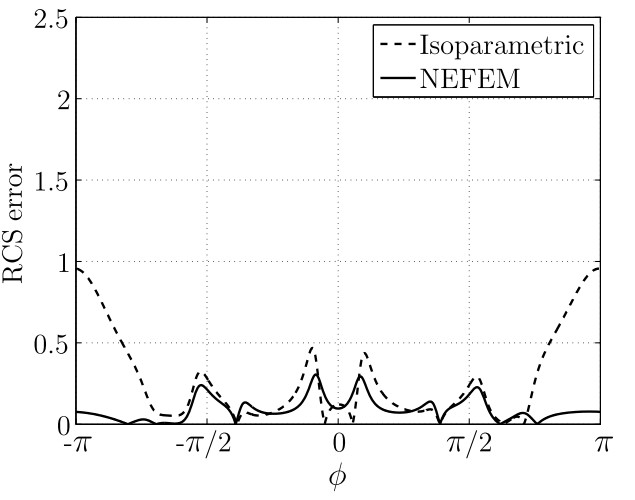

(b) $p=6$

Fig. 71 Scattering by a PEC sphere of diameter $\lambda$

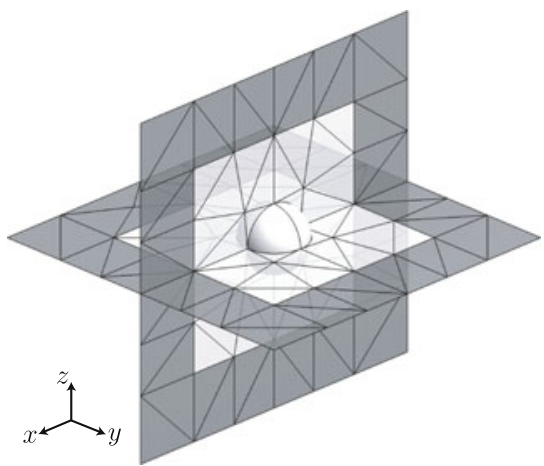

(a) Mesh

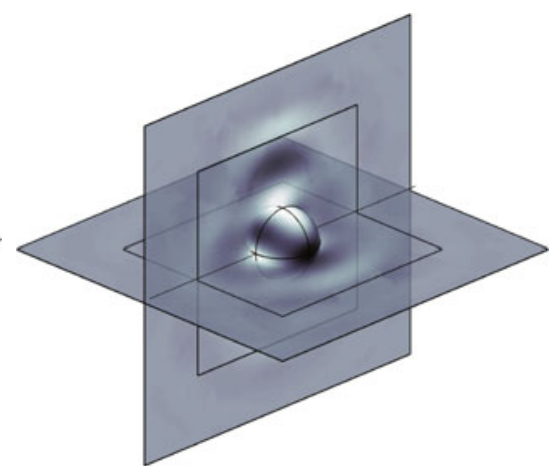

(b) $E_{1}$ field
Figure 70 compares the RCS error distribution with isoparametric FEs and NEFEM, for a degree of approximation $p=5$ and $p=6$. Note that the maximum error with isoparametric FEs is observed at viewing angles corresponding to the leading edge $(\phi=-\pi$ and $\phi=\pi)$, whereas for NEFEM the maximum error is obtained near the singularity $(\phi=0)$.

This example illustrates the sensitivity of the RCS to poor geometric representations. Isoparametric approximations are not sufficient when coarse meshes and high-order approximations are considered. Geometric errors may lead to important discrepancies in the RCS. Thus, $h$-refinement is usually performed at the leading edge of airfoils to provide an accurate representation of the geometry. With NEFEM, the exact boundary representation allows to mesh the domain with no dependence on the geometrical complexity. Using only one element per wavelength and $p=5$, i.e. 6 nodes per wavelength, an accurate solution is obtained, without performing $h$-refinement. The maximum error in a NEFEM computations is observed where the solution is complex, not where the geometry is complex.

Next example considers an incident plane wave traveling in the $z^{+}$direction and scattered by a PEC sphere of diameter $\lambda$. The sphere is exactly described with a quadratic singular NURBS surface, and a coarse mesh with only eight elements for the discretization of the curved boundary is considered, see two cuts of the volume mesh and the surface mesh of the sphere in Fig. 71(a). The mesh has 1271 elements with planar faces and 32 curved elements ( 8 elements with a face on the NURBS boundary and 24 elements with an edge on the NURBS boundary). Scattered $E_{1}$ field computed with NEFEM and a polynomial approximation of degree $p=5$ is represented in Fig. 71(b), showing the field intensity on the sphere surface and illustrating the absorption of outgoing waves in the PML.

Figures 72 and 73 compare the RCS computed with degree $p=3$ and $p=4$ with the analytical solution, for vertical and horizontal polarization respectively. For Cartesian FEs, the RCS error is not reduced for all viewing angles as $p$ increases. In particular, the RCS near viewing angles $-\pi$ and $\pi$ is more accurate with $p=3$ than using $p=4$, see Figs. 72(a) and 73(a). Again, the approximate boundary representation has a critical influence in the RCS. NEFEM exhibits the same robustness than in the previous examples. The error is decreased for all viewing angles as the degree of the approximation is increased. A perfect match between analytical and computed solution is observed with $p=4$, see Figs. 72(b) and 73(b).

Note that Cartesian FEs offer a slightly different performance for vertical and horizontal polarizations. In fact, 
Fig. 72 Scattering by a PEC sphere of diameter $\lambda$ : RCS comparison for increasing $p$ and for the vertical polarization

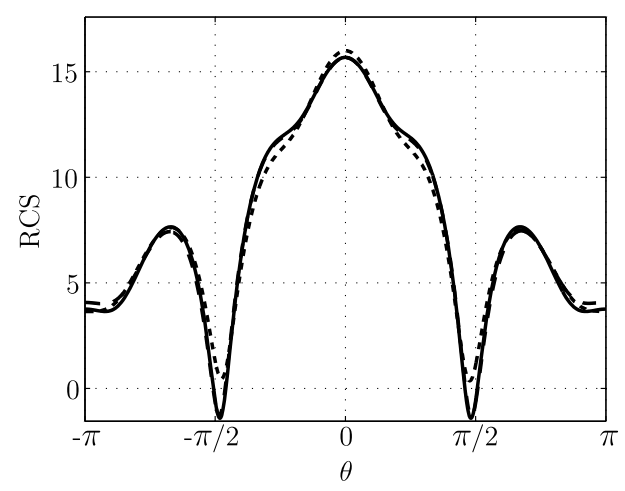

(a) Cartesian FEM

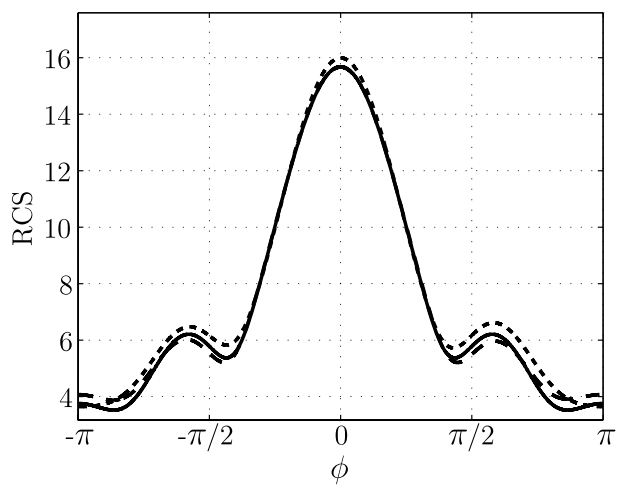

(a) Cartesian FEM

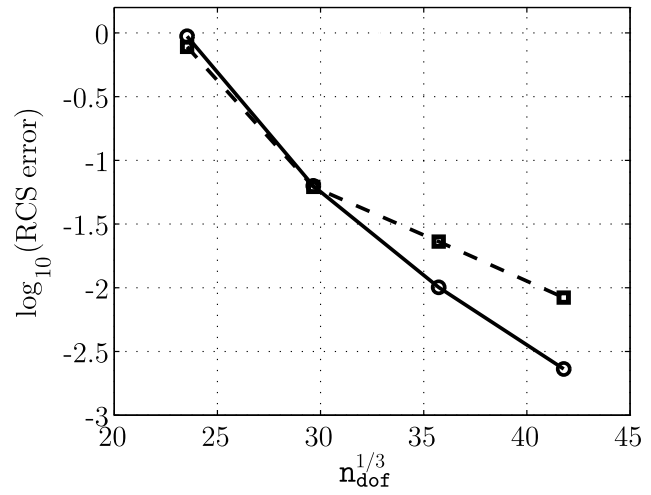

(a) Vertical polarization

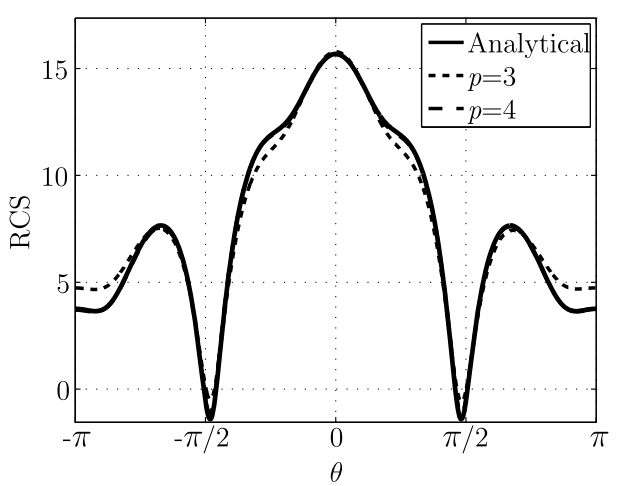

(b) NEFEM

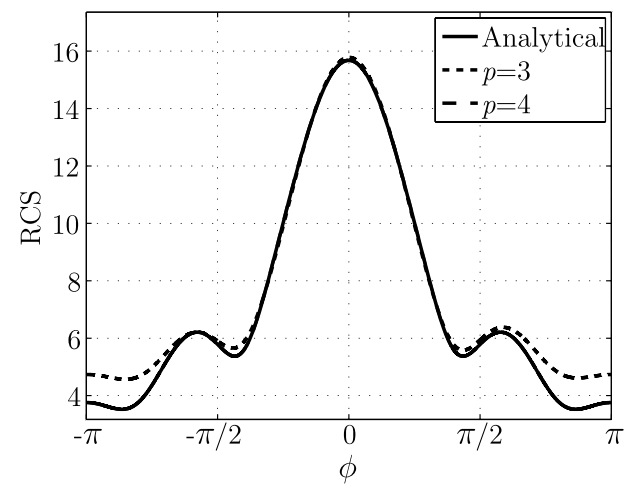

(b) NEFEM

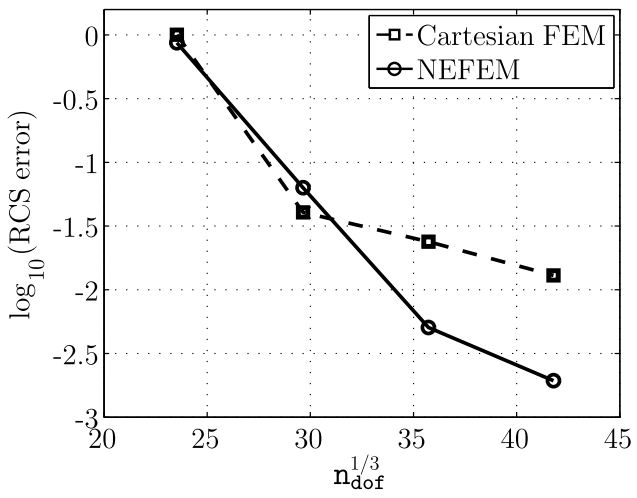

(b) Horizontal polarization
Fig. 74 Scattering by a PEC sphere of diameter $\lambda$ : $p$-convergence comparison of the RCS error
Fig. 73 Scattering by a PEC comparison for increasing $p$ and for the horizontal polarization higher errors are observed for the horizontal polarization, whereas for NEFEM, almost identical performance is observed for both polarizations. To compare accuracy, Fig. 74 represents the RCS error in the $\mathcal{L}^{2}(-\pi, \pi)$ norm for increasing $p$, starting with $p=2$, showing the superiority of NEFEM compared to Cartesian FEs. The most critical difference is observed in the horizontal polarization for $p=5$. NEFEM is almost one order of magnitude more precise than the corresponding Cartesian FEs. It is worth remarking that the RCS error for Cartesian FEs is controlled by the geometric error for $p>3$. In fact, the isoparametric approximation of the sphere with 8 curved elements is considered in
Sect. 6.1, and a similar performance is observed in a secondorder elliptic problem.

Compared to other techniques, NEFEM is also more accurate and efficient. For instance, to achieve an accuracy of $10^{-2}$ measuring the maximum norm of the scattering width, more than 100000 degrees of freedom are required using high-order edge elements [60]. With NEFEM, a degree of approximation $p=4$ provides an error of $4.7 \times 10^{-3}$, using 45605 degrees of freedom, that is, NEFEM is two times more accurate by using $50 \%$ of the $\mathrm{n}_{\mathrm{dof}}$, showing that NEFEM is also competitive in 3D compared to other techniques used by the CEM community. 
Fig. 81 Scattering by a PEC NASA almond of characteristic length $8 \lambda$ : RCS for a NEFEM solution with $p=5$

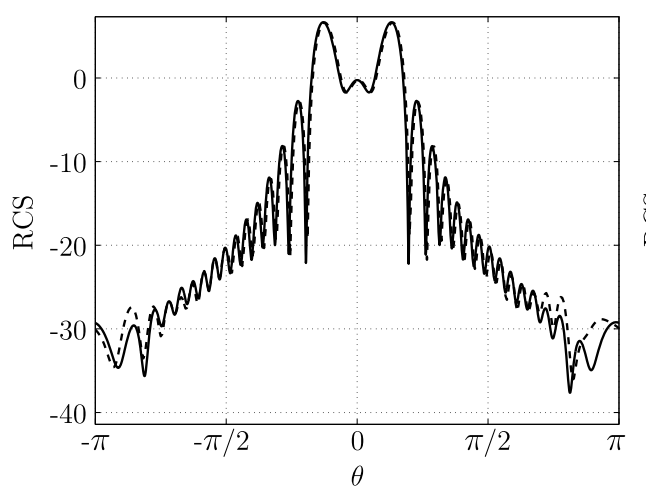

(a) Vertical polarization

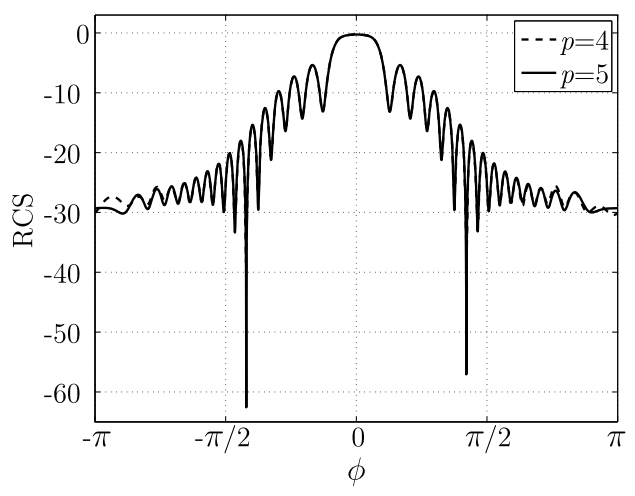

(b) Horizontal polarization
Fig. 82 Scattering by a PEC NASA almond of characteristic length $21 \lambda$ : surface mesh on the almond and two components of the scattered field for a NEFEM solution with $p=3$

Fig. 83 Scattering by a PEC NASA almond of characteristic length $21 \lambda$ : RCS for a NEFEM solution with $p=4$

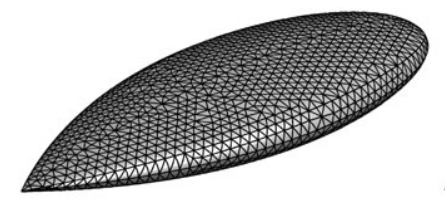

(a) Surface mesh

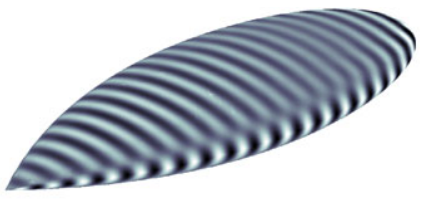

(b) $E_{3}$

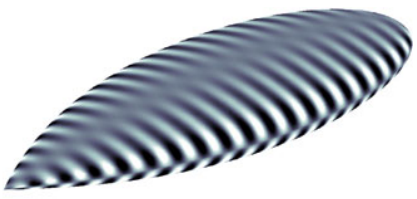

(c) $H_{3}$

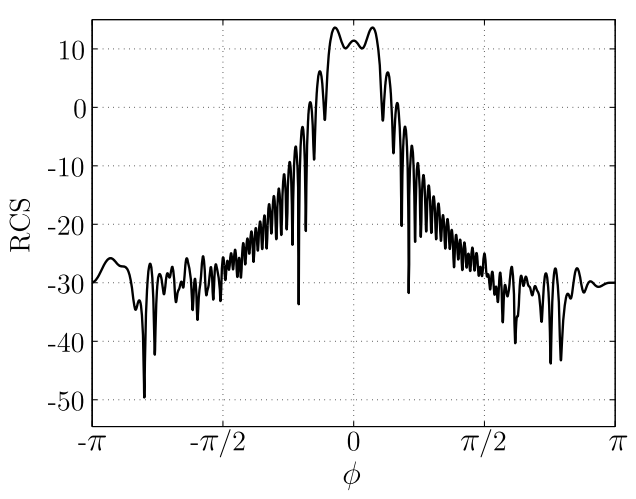

(a) Vertical polarization

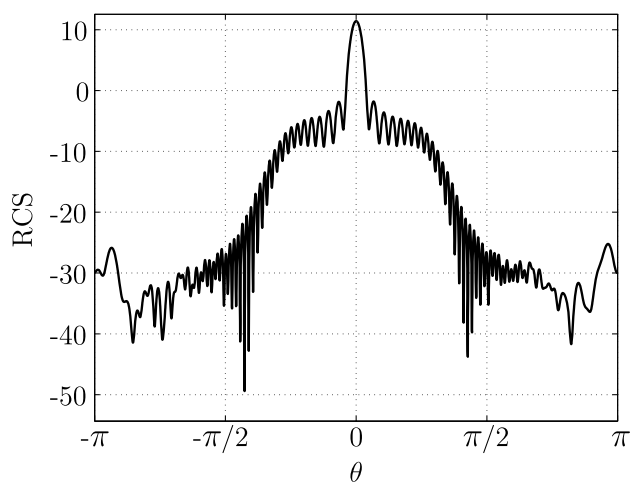

(b) Horizontal polarization
Figure 81 shows the RCS for vertical and horizontal polarizations. Two RCS patterns are displayed, for a NEFEM solution with $p=4$ and $p=5$ respectively. Results show a perfect agreement with published results [42], which are obtained with linear FEs in a tetrahedral mesh with 1121431 mesh nodes. Thus, this example shows the competitiveness of NEFEM compared to other formulations for more challenging applications. Even if a DG formulation is considered, i.e. duplicating nodes at inter-element faces, the computation requires less degrees of freedom to obtain similar accuracy, due to the good performance of NEFEM with coarse meshes and high-order approximations.

Next, the scattering by a PEC NASA almond of characteristic length $21 \lambda$ is considered. The mesh has 48699 elements with planar faces and 6008 curved elements. Figure 82 shows the surface mesh on the almond and two components of the scattered field computed with NEFEM and $p=3$, corresponding to a wave incident onto the tip of the almond. RCS distribution for vertical and horizontal polarization are represented in Fig. 83. Results compare well with published results [42], and again show the competitiveness of NEFEM for higher frequency problems. The tetrahedral mesh used in [42] has 51342008 linear elements, and approximately 8 million of nodes. With NEFEM and $p=4$ the mesh has 2 million of nodes (including the duplication due to the DG formulation), requiring four times less degrees of freedom than using standard linear FEs.

The results presented in this section has shown the benefits of NEFEM using standard FE meshes. However, the possibilities of NEFEM still go beyond.

It is well known that, in the context of FEs, the size of the model is sometimes subsidiary of the geometrical complexity and not only on solution itself. In particular, FE simulation of the scattering by complex objects with small geometric details requires drastic $h$-refinement to capture the geometry. Moreover, for scattering applications, small ge- 


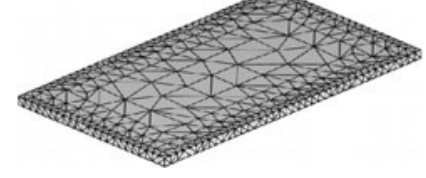

(a) Reference FEM mesh

Fig. 88 Scattering by a PEC thin plate: standard FE meshes

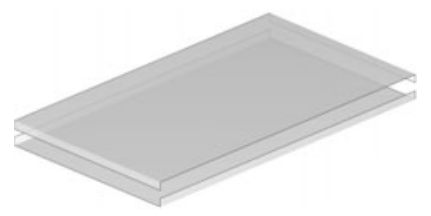

(a) NURBS surfaces

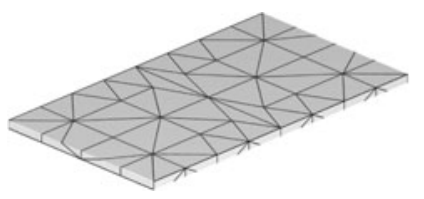

(b) NEFEM mesh
Fig. 89 Scattering by a PEC thin plate: NURBS surfaces (separated for visualization) and NEFEM coarse mesh with elements containing edge singularities

in Fig. 84, induces important differences in the time-step size when explicit time integrators are used and, therefore, adds another advantage of NEFEM. In the refined mesh with $p=5$ the minimum distance between two mesh nodes is $1.4 \times 10^{-5}$, whereas in the NEFEM coarse mesh with $p=12$ the minimum distance is $1.2 \times 10^{-3}$. The computation with the refined mesh requires 527459 time steps, whereas the computation in the coarse NEFEM mesh requires 6620 time steps.

The aim of the last example is to show the possibilities of NEFEM elements, containing edge singularities, in 3D domains. In large scale 3D computations, very small geometric details may lead to unaffordable computational times with explicit time-marching algorithms, due to the excessive $h$-refinement needed to accurately capture the geometry.

To show the capabilities of NEFEM in this scenario, the scattering by a PEC thin plate of dimensions $\lambda \times 4 \lambda / 7 \times$ $\lambda / 22$ is considered. The small thickness of the plate, with respect to the wave length $\lambda$, implies that $h$-refinement in standard FE meshes is controlled by the thickness of the plate, not by the desired number of nodes per wavelength.

Two standard FEM computational meshes are considered to compare the accuracy of NEFEM computations. Figure 88(a) shows a standard FE mesh with refinement towards the singularities of the plate. The second mesh, in Fig. 88(b), is a FEM mesh with a desired mesh size of about $\lambda / 8$. As usual, a standard mesh generator needs to perform extra $h$ refinement to offer an accurate description of the geometrical model. Therefore, the minimum mesh size in a standard FE mesh is, at least, $\lambda / 22$.

Nevertheless, the mesh size for NEFEM is not controlled by small geometric features, and the desired mesh size is maintained, even in the presence of singularities in the boundary of the domain. The plate is exactly represented by two NURBS surfaces with $\mathcal{C}^{0}$ continuity at the edges of the

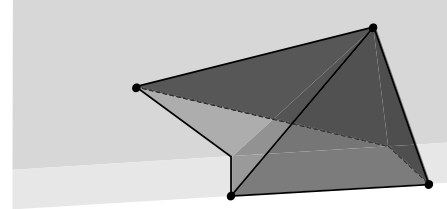

Fig. 90 Detailed view of a NEFEM element containing an edge singularity in its boundary face

plate, as illustrated in Fig. 89(a). A NEFEM coarse mesh is represented in Fig. 89(b). Note that, to obtain the desired mesh size, some elements contain an edge singularity inside one NURBS face, see a detailed view of a NEFEM element in Fig. 90.

Figure 91 compares the RCS distribution for vertical and horizontal polarizations. An excellent agreement is observed between the three computations, showing the potential of NEFEM coarse meshes with elements containing singularities. Again, the maximum disagreement is obtained at singularities of the RCS due to its logarithmic scale. The error of the scattering width and in the $\mathcal{L}^{2}(-\pi, \pi)$ norm for NEFEM is $3.2 \times 10^{-2}$ and $4.7 \times 10^{-2}$ for the vertical and horizontal polarizations respectively. Despite the known limitation of polynomial approximation for the approximation of singular solutions it is remarkable the quality obtained in the RCS patterns by using NEFEM meshes.

\section{Closing Remarks}

This papers presents a complete overview of recently proposed NURBS-enhanced finite element method (NEFEM). This methodology is an improvement of the standard FEM where the exact CAD description of the geometrical model is considered, but only for the boundary of the computational domain. At elements intersecting the NURBS boundary specific strategies to perform the interpolation and the numerical integration are proposed. NEFEM defines the approximation directly with Cartesian coordinates, ensuring reproducibility of polynomials in the physical space. The key idea of the numerical integration technique is to use specifically designed mappings for curved elements in order to decouple complexity of the NURBS boundary description, allowing a seamless treatment of trimmed and singular NURBS surfaces in 3D. It is worth recalling that at elements not intersecting the boundary classical FE are used, preserving the efficiency of the FEM.

The application and superiority of NEFEM with respect to other curved FE techniques has been presented. Ranging from second-order elliptic problems solved with a standard continuous Galerkin formulation to more complex applications such as the numerical solution of Euler and Maxwell's 
7. Barth T, Ohlberger M (2004) Finite volume methods: Foundation and analysis. In: Stein E, de Borst, R, Hughes TJR (eds) Fundamentals, encyclopedia of computational mechanics, vol 1. Wiley, New York. Chap 4

8. Barth TJ (1998) Simplified numerical methods for gas dynamics systems on triangulated domains. PhD thesis, Stanford University

9. Bassi F, Rebay S (1997) High-order accurate discontinuous finite element solution of the 2D Euler equations. J Comput Phys 138(2):251-285

10. Bazilevs Y, Hughes TJR (2007) Weak imposition of Dirichlet boundary conditions in fluid mechanics. Comput Fluids 36(1):12-26

11. Berenger JP (1994) A perfectly matched layer for the absorption of electromagnetic waves. J Comput Phys 114(2):185-200

12. Bernardi C (1989) Optimal finite-element interpolation on curved domains. SIAM J Numer Anal 26(5):1212-1240

13. Brenner SC, Scott LR (1994) The mathematical theory of finite element methods. Springer, Berlin

14. Cavendish JC, Gordon WJ, Hall CA (1976) Ritz-Galerkin approximations in blending function spaces. Numer Math 26(2):155-178

15. Chen Q, Babuška I (1995) Approximate optimal points for polynomial interpolation of real functions in an interval and in a triangle. Comput Methods Appl Mech Eng 128(3-4):405-417

16. Chen Q, Babuška I (1996) The optimal symmetrical points for polynomial interpolation of real functions in the tetrahedron. Comput Methods Appl Mech Eng 137(1):89-94

17. Ciarlet PG, Raviart PA (1972) Interpolation theory over curved elements with applications to finite element methods. Comput Methods Appl Mech Eng 1(1):217-249

18. Cirak F, Ortiz M, Schröder J (2000) Subdivision surfaces: a new paradigm for thin-shell finite-element analysis. Int $\mathrm{J}$ Numer Methods Eng 47(12):2039-2072

19. Clough RW (2004) Early history of the finite element method from the view point of a pioneer. Int J Numer Methods Eng 60(1):283-287

20. Cockburn B (2004) Discontinuous Galerkin methods for computational fluid dynamics. In: Stein E, de Borst R, Hughes TJR (eds) Fluids. Encyclopedia of computational mechanics, vol 3. Wiley, New York. Chap 4

21. Cockburn B, Shu CW (1989) TVB Runge-Kutta local projection discontinuous Galerkin finite element method for conservationlaws II. General framework. Math Comput 52(186):411-435

22. Cockburn B, Karniadakis GE, Shu CW (2000) The development of discontinuous Galerkin methods. In: Cockburn B, Karniadakis GE, Shu CW (eds) Discontinuous Galerkin methods. Lecture notes in computational science and engineering, vol 11. Springer, Berlin, pp 3-50

23. Cockburn B, Karniadakis GE, Shu CW (2000) The development of discontinuous Galerkin methods. In: Cockburn B, Karniadakis GE, Shu CW (eds) Discontinuous Galerkin methods. Springer, Berlin

24. Costabel M, Dauge M (1997) Singularities of electromagnetic fields in polyhedral domains. Arch Ration Mech Anal 151(3):221-276

25. Cottrell JA, Hughes TJR, Bazilevs Y (2009) Isogeometric analysis: Toward integration of CAD and FEA. Wiley, New York

26. Coyle J, Ledger PD (2005) Evidence of exponential convergence in the computation of Maxwell eigenvalues. Comput Methods Appl Mech Eng 194(2-5):587-604

27. Dadone A, Grossman B (1994) Surface boundary conditions for the numerical solution of the Euler equations. AIAA J 32(2):285-293

28. Davis P, Rabinowitz P (1984) Methods of numerical integration, 2nd edn. Academic Press, New York
29. Dey S, Shephard MS, Flaherty JE (1997) Geometry representation issues associated with $p$-version finite element computations. Comput Methods Appl Mech Eng 150(1-4):39-55

30. Dominek AK, Shamanski HT (1990) The almond test body. The Ohio State University ElectroScience Laboratory, Department of Electrical Engineering, Report 721929-9, NASA Langley Research Center

31. Donea J, Huerta A (2005) Finite element methods for flow problems. Wiley, New York

32. Dorr MR (1984) The approximation-theory for the $p$-version of the finite-element method. SIAM J Numer Anal 21(6):1180 1207

33. Dubiner M (1991) Spectral methods on triangles and other domains. J Sci Comput 6:345-390

34. Dumbser M, Munz CD (2007) On source terms and boundary conditions using high order arbitrary discontinuous Galerkin schemes. Int J Appl Math Comput Sci 17(3):297-310

35. Ergatoudis J, Irons BM, Zienkiewicz OC (1968) Curved isoparametric "quadrilateral" elements for finite element analysis. Int J Solids Struct 4(1):31-42

36. Fernández-Méndez S, Huerta A (2004) Imposing essential boundary conditions in mesh-free methods. Comput Methods Appl Mech Eng 192(12-14):1257-1275

37. Gao H, Wang ZJ, Liu Y (2010) A study of curved boundary representations for 2D high order Euler solvers. J Sci Comput 44(3):323-336

38. Gordon WJ, Hall CA (1973) Construction of curvilinear coordinate systems and applications to mesh generation. Int J Numer Methods Eng 7(4):461-477

39. Gordon WJ, Hall CA (1973) Transfinite element methods: Blending-function interpolation over arbitrary curved element domains. Numer Math 21(2):109-129

40. Gottlieb S, Shu CW (1998) Total variation diminishing RungeKutta schemes. Math Comput 67(221):73-85

41. Gui W, Babuška I: (1986) The $h$-version, $p$-version and $h$ - $p$ version of the finite-element method in 1-dimension. 1 The error analysis of the $p$-version. Numer Math 49(6):577-612

42. Hachemi ME, Hassan O, Morgan K, Rowse D, Weatherill N (2004) A low-order unstructured-mesh approach for computational electromagnetics in the time domain. Philos Trans R Soc Lond Ser A Math Phys Eng Sci 362(1816):445-469

43. Harrington RF (1961) Time-harmonic electromagnetic fields. McGraw-Hill, New York

44. Harris R, Wang ZJ, Liu Y (2007) Efficient implementation of high-order spectral volume method for multidimensional conservation laws on unstructured grids. In: 45th AIAA aerospace sciences meeting and exhibit. AIAA, Nevada

45. Hesthaven JS (2000) Stable spectral methods on tetrahedral elements. SIAM J Numer Anal 21(6):2352-2380

46. Hesthaven JS, Warburton T (2002) Nodal high-order methods on unstructured grids I. time-domain solution of Maxwell's equations. J Comput Phys 181(1):186-221

47. Hirsch C (1988) Numerical computation of internal and external flows. Fundamentals of numerical discretization, vol 1. Wiley, New York

48. Huerta A, Rodríguez-Ferran A, Díez P, Sarrate J (1999) Adaptive finite element strategies based on error assessment. Int J Numer Methods Eng 46:1803-1818

49. Hughes TJR, Cottrell JA, Bazilevs Y (2005) Isogeometric analysis: CAD finite elements, NURBS, exact geometry and mesh refinement. Comput Methods Appl Mech Eng 194(39-41):41354195

50. Huttunen T, Malinen M, Monk P (2007) Solving Maxwell's equations using the ultra weak variational formulation. J Comput Phys 223(2):731-758

51. Inoue K, Kikuchi Y, Masuyama T (2005) A NURBS finite element method for product shape design. J Eng Des 16(2):157-174 
52. Johnson C (1987) Numerical solution of partial differential equations by the finite element method. Cambridge University Press, Cambridge

53. Kagan P, Fischer A, Bar-Yoseph PZ (1998) New B-spline finite element approach for geometrical design and mechanical analysis. Int J Numer Methods Eng 41(3):435-458

54. Karniadakis GE, Sherwin SJ (1999) Spectral $/ h p$ element methods for CFD (Numerical mathematics and scientific computation). Oxford University Press, Oxford

55. Krivodonova L, Berger M (2006) High-order accurate implementation of solid wall boundary conditions in curved geometries. J Comput Phys 211(2):492-512

56. Ladson CL, Brooks CW, Hill AS, Sproles DW (1996) Computer program to obtain ordinates for naca airfoils. Tech Rep NASA TM-4741. NASA Langley Research Center. http://techreports.larc.nasa.gov/ltrs/PDF/NASA-96-tm4741.pdf

57. Landmann B, Kessler M, Wagner S, Krämer E (2008) A parallel high-order discontinuous Galerkin code for laminar and turbulent flows. Comput Fluids 37(4):427-438

58. Laney CB (1998) Computational gasdynamics. Cambridge University Press, Cambridge

59. Ledger PD, Morgan K, Hassan O (2007) Electromagnetic scattering simulation using an $\mathbf{H}$ (curl) conforming $h p$ finite element method in three dimensions. Int J Numer Methods Fluids 53(8):1267-1296

60. Ledger PD, Morgan K, Hassan O, Weatherill NP (2003) Plane wave $\mathbf{H}(\mathrm{curl}, \Omega$ ) conforming finite elements for Maxwell's equations. Comput Mech 31(3-4):272-283

61. Lenoir M (1986) Optimal isoparametric finite elements and error estimates for domains involving curved boundaries. SIAM J Numer Anal 23(3):562-580

62. Luhon C, Wagner S (2007) Three-dimensional discontinuous Galerkin codes to simulate viscous flow by spatial discretization of high order and curved elements on unstructured grids. In: New results in numerical and experimental fluid mechanics VI. Springer, Berlin, pp 145-153

63. Luo H, Pozrikidis C (2006) A Lobatto interpolation grid in the tetrahedron. IMA J Appl Math 71(2):298-313

64. Luo XJ, Shephard MS, Remacle JF (2001) Influence of geometric approximation on the accuracy of higher order methods. Tech Rep 1, SCOREC

65. Lyness JN, Jespersen D (1975) Moderate degree symmetric quadrature rules for the triangle. IMA J Appl Math 15:19-32

66. Ma YL, Hewitt WT (2003) Point inversion and projection for NURBS curve and surface: control polygon approach. Comput Aided Geom Des 20:79-99

67. Mäkipelto J (2004) Geometry based rational enrichment functions for triangular plane elasticity element. In: Proceedings of the 21st international congress of theoretical and applied mechanics. Poland

68. McLeod R (1979) A piecewise parabolic $\mathcal{C}^{1}$ approximation technique for curved boundaries. Comput Math Appl 5:277-284

69. Muñoz JJ (2008) Modelling unilateral frictionless contact using the null-space method and cubic B-spline interpolation. Comput Methods Appl Mech Eng 197(9-12):979-993

70. Nitsche JA (1971) Über ein variations zur lösung von dirichletproblemen bei verwendung von teilräumen die keinen randbedingungen unterworfen sind. Abh Math Semin Univ Hamb 36:9-15

71. Pascal JF, George PL (1999) Maillages : applications aux éléments finis. Hermès, Paris

72. Piegl L, Tiller W (1995) The NURBS book. Springer, London

73. Raviart PA, Thomas JM (1998) Introduction à l'analyse numérique des équations aux dérivées partielles. Dunod, Paris

74. Rogers DF (2001) An introduction to NURBS with historical perspective. Academic Press, San Diego
75. Schramm U, Pilkey W (1993) The coupling of geometric descriptions and finite elements using NURBS: a study of shape optimization. Finite Elem Anal Des 15(1):11-34

76. Schuh MJ, Woo AC, Simon MP (1994) The monostatic/bistatic approximation. IEEE Trans Antennas Propag Mag 35(4):76-78

77. Scott LR (1973) Finite element techniques for curved boundaries. PhD thesis, Massachusetts Institute of Technology, Dept of Mathematics

78. Scott LR (1975) Interpolated boundary conditions in the finite element method. SIAM J Numer Anal 12(3):404-427

79. Sevilla R, Fernández-Méndez S, Huerta A (2008) NURBSenhanced finite element method (NEFEM). Int J Numer Methods Eng 76(1):56-83

80. Sevilla R, Fernández-Méndez S (2011) Numerical integration over 2D NURBS shaped domains with applications to NURBSenhanced FEM. Finite Elem Anal Des 47(10):1209-1220

81. Sevilla R, Fernández-Méndez S, Huerta A (2008) NURBSenhanced finite element method (NEFEM) for Euler equations. Int J Numer Methods Fluids 57(9):1051-1069

82. Sevilla R, Fernández-Méndez S, Huerta A (2011) 3D-NURBSenhanced finite element method (NEFEM). Int J Numer Methods Eng 88(2):103-125

83. Sevilla R, Fernández-Méndez S, Huerta A (2011) Comparison of high-order curved finite elements. Int J Numer Methods Eng 87(8):719-734

84. Sherwin SJ, Karniadakis G (1995) A new triangular and tetrahedral basis for high-order $(h p)$ finite element methods. Int $\mathrm{J} \mathrm{Nu}-$ mer Methods Eng 38(22):3775-3802

85. Solin P, Segeth K (2003) Higher-order finite element methods. Chapman \& Hall, London

86. Szabó B, Babuška I (1991) Finite element analysis. Wiley, New York

87. Szabó B, Düster A, Rank E (2004) The $p$-version of the finite element method. Encyclopedia of computational mechanics, vol 1 (Fundamentals). Wiley, New York. Chap 5

88. Szegö G (1975) Orthogonal polynomials, 4th edn. American Mathematical Society, Providence

89. Taflove A (1995) Computational electrodynamics: The finitedifference time-domain method. Artech House, Inc, Norwood

90. Taylor MA, Wingate BA, Vincent RE (2000) An algorithm for computing Fekete points in the triangle. SIAM J Numer Anal 38(5):1707-1720

91. Toro EF (1997) Riemann solvers and numerical methods for fluid dynamics. Springer, Berlin

92. Turkel E, Yefet A (1998) Absorbing PML boundary layers for wave-like equations. Appl Numer Math 27(4):533-557

93. Van der Ven H, Van der Vegt JJW (2002) Space-time discontinuous Galerkin finite element method with dynamic grid motion for inviscid compressible flows II. Efficient flux quadrature. Comput Methods Appl Mech Eng 191(41-42):4747-4780

94. Wachspress EL (1973) A rational basis for function approximation. II Curved sides. J Inst Math Appl 11(1):83-104

95. Wachspress EL (1975) A rational finite element basis, vol 114. Academic Press, New York

96. Wachspress EL (1981) High-order curved finite elements. Int J Numer Methods Eng 17(5):735-745

97. Wandzura S, Xiao H (2003) Symmetric quadrature rules on a triangle. Comput Math Appl 45(12):1829-1840

98. Wang ZJ, Liu Y (2006) Extension of the spectral volume method to high-order boundary representation. J Comput Phys 211(1):154-178

99. Wang ZJ, Sun Y (2002) A curvature-based wall boundary condition for the Euler equations on unstructured grids. In: Proceedings of the 40th AIAA aerospace sciences meeting and exhibit. AIAA, Nevada 
100. Wang ZJ, Zhang L, Liu Y (2004) Spectral (finite) volume method for conservation laws on unstructured grids IV: extension to twodimensional Euler equations. J Comput Phys 194(2):716-741

101. Warburton T (2006) An explicit construction of interpolation nodes on the simplex. J Eng Math 56(3):247-262

102. Woo AC, Wang HTG, Schub MJ (1993) Benchmark radar targets for the validation of computational electromagnetics programs. IEEE Trans Antennas Propag Mag 35(1):84-89

103. Xue D, Demkowicz L (2005) Control of geometry induced error in $h p$ finite element (FE) simulations. I Evaluation of FE error for curvilinear geometries. Int J Numer Anal Model 2(3):283-300

104. Zienkiewicz OC (1971) The finite element method in engineering science, 2nd edn. McGraw-Hill, London
105. Zienkiewicz OC (1995) Origins milestones and directions of the finite element method-A personal view. Arch Comput Methods Eng 2(1): 1-48

106. Zienkiewicz OC, Taylor RL (2000) The finite element method, vol 1. The basis, 5th edn. Butterwort-Heinemann, Stoneham

107. Zlámal M (1973) Curved elements in the finite element method. I. SIAM J Numer Anal 10(1):229-240

108. Zlámal M (1973) The finite element method in domains with curved boundaries. Int J Numer Methods Eng 5(3):367-373

109. Zlámal M (1974) Curved elements in the finite element method. II. SIAM J Numer Anal 11(2):347-362 